\title{
Linear Stability Analysis for a Reactive, Multiphase Bubble Column
}

Justin D. Smith, Daniel W. Crunkleton ${ }^{*}$, Selen Cremaschi

Russell School of Chemical Engineering, The University of Tulsa, 800 S Tucker Drive, Tulsa, OK 74104, United States

* daniel-crunkleton@utulsa.edu 


\section{Equation S1}

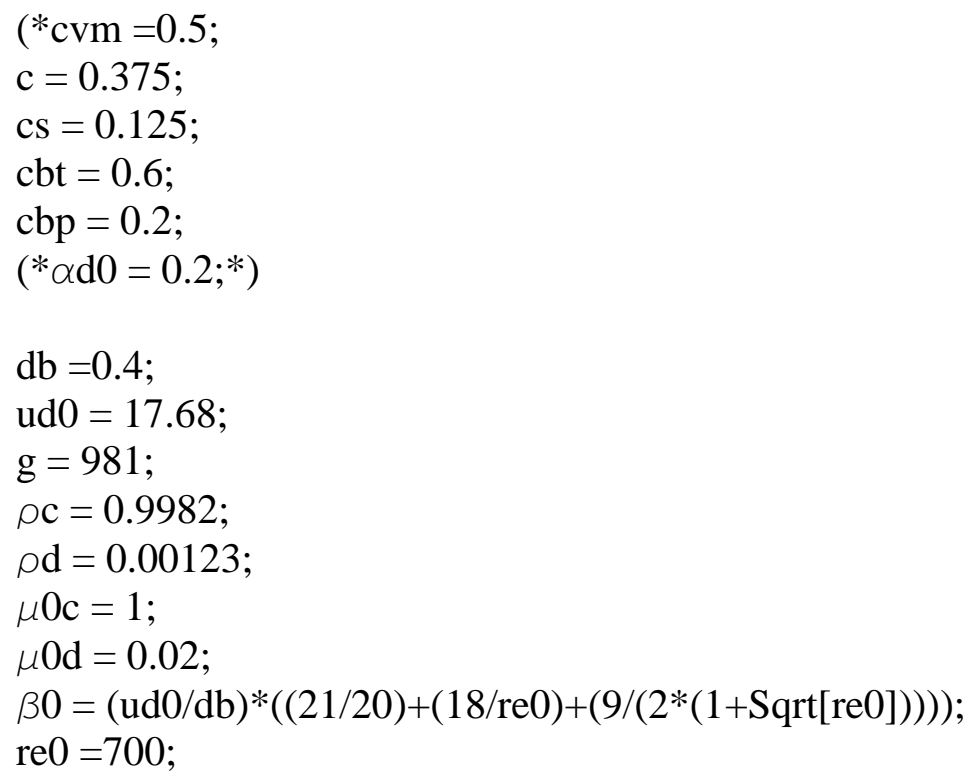

$\beta 1=(\mathrm{ud} 0 / \mathrm{db}) *((21 / 20)+(18 / \mathrm{re} 0)+(9 /(1+\operatorname{Sqrt}[\mathrm{re} 0]))-$

$((9 * \operatorname{Sqrt}[\mathrm{re} 0]) /(4 *((1+\operatorname{Sqrt}[\mathrm{re} 0]) *(1+\operatorname{Sqrt}[\mathrm{re} 0])))))$;

$\alpha \mathrm{dcp}=1$

$\left.\rho \mathrm{v} 0=(1-\alpha \mathrm{d} 0)^{*} \rho \mathrm{c}+\alpha \mathrm{d} 0 * \rho \mathrm{d} ; *\right)$

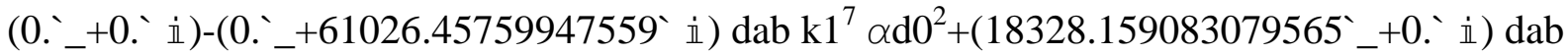

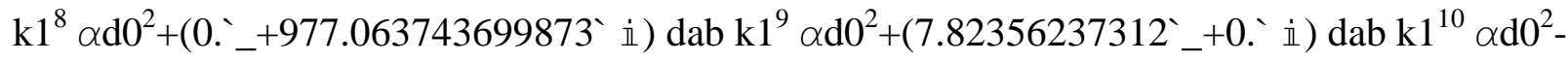
$\left(0 .{ }_{-}+183079.37279842678\right.$ i $) \mathrm{dab} \mathrm{k} 1^{5} \mathrm{k} 2^{2} \alpha \mathrm{d} 0^{2}+\left(54886.74736522122^{\prime}+0\right.$.

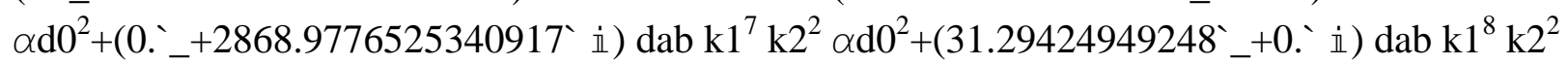

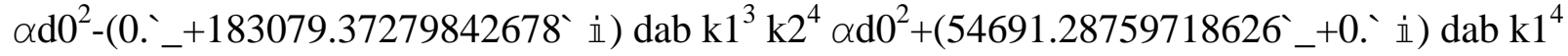

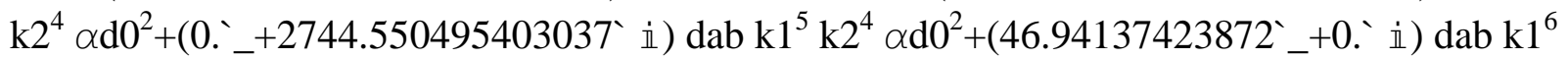

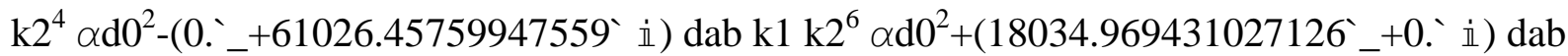

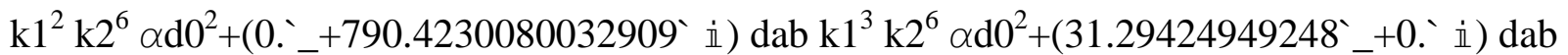

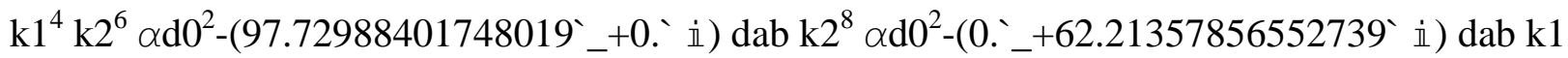

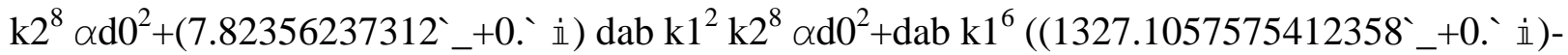
(1327.1057575412358__+0`立) $\alpha \mathrm{d} 0) \alpha \mathrm{d} 0^{3}+\mathrm{dab} \mathrm{k}^{2} \mathrm{k} 2^{4} \alpha \mathrm{d} 0^{3}\left(\left(-2.0160335991377404{ }^{` *} 6+0\right.\right.$. i) $+\left(\left(4.030908475875317^{* \wedge} 6+0\right.\right.$.`立 $)-\left(2.014874876737577^{` * \wedge} 6+0\right.$.`立 $\left.\left.) \alpha \mathrm{d} 0\right) \alpha \mathrm{d} 0\right)+\mathrm{dab} \mathrm{k} 1^{4} \mathrm{k} 2^{2}$ $\alpha \mathrm{d} 0^{3}\left(\left(-1.0060261409325583^{` * \wedge} 6+0\right.\right.$. $\left.\dot{\mathbb{1}}\right)+\left(\left(2.0134635793013468^{` * \wedge} 6+0\right.\right.$. $\left.\dot{\mathbb{1}}\right)-$ $\left(1.0074374383687885^{* \wedge} 6+0\right.$. $\left.\left.\left.\dot{\mathbb{1}}\right) \alpha \mathrm{d} 0\right) \alpha \mathrm{d} 0\right)+\mathrm{dab} \mathrm{k} 2^{6} \alpha \mathrm{d} 0^{3}\left(\left(-1.0086803524476408^{` * \wedge} 6+0\right.\right.$.'

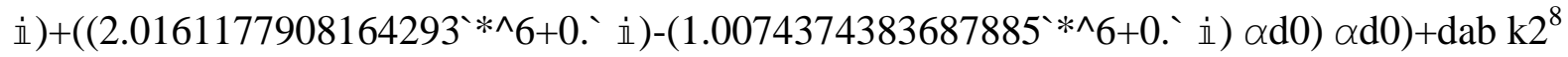
$\alpha \mathrm{d} 0^{3}\left(\left(-16215.369143931362 `+0\right.\right.$ ` $\left.^{\mathrm{i}}\right)+\alpha \mathrm{d} 0\left(\left(-59665.1516927372 `+0\right.\right.$. $^{\prime}$

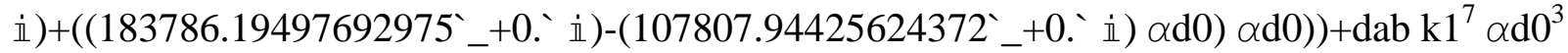

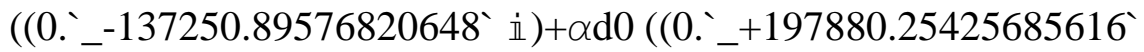

$\dot{\mathbb{1}})+\left(\left(0 .{ }_{-}+476.40438894664044\right.\right.$ i $\left.\left.\left.)-\left(0 .{ }_{-}+79.30527812076777^{\prime} \dot{\mathbb{1}}\right) \alpha \mathrm{d} 0\right) \alpha \mathrm{d} 0\right)\right)+\mathrm{dab} \mathrm{k} 1^{2} \mathrm{k} 2^{8}$ 


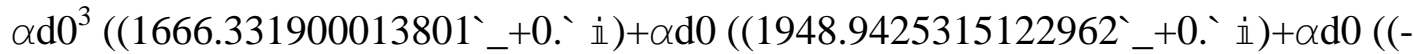

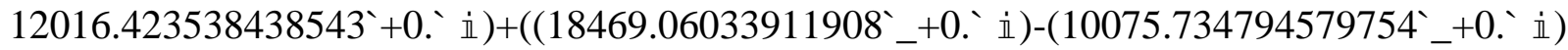
$\alpha \mathrm{d} 0) \alpha \mathrm{d} 0)))+\mathrm{dab} \mathrm{k} 1^{4} \mathrm{k} 2^{6} \alpha \mathrm{d} 0^{3}\left(\left(6690.289180189604{ }_{-}+0\right.\right.$.` $\left.^{\mathrm{i}}\right)+\alpha \mathrm{d} 0$

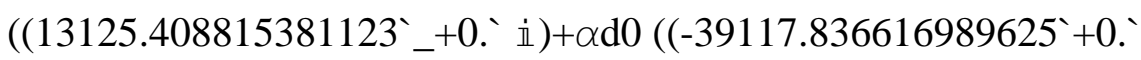
$\left.\left.\left.\dot{\mathbb{i}})+\left(\left(25988.000901646257{ }_{-}+0{ }^{`} \dot{\mathbb{1}}\right)-\left(6717.156529719836{ }_{-}+0{ }^{`} \dot{\mathbb{1}}\right) \alpha \mathrm{d} 0\right) \alpha \mathrm{d} 0\right)\right)\right)+\mathrm{dab} \mathrm{k} 2{ }^{10} \alpha \mathrm{d} 0^{3}$

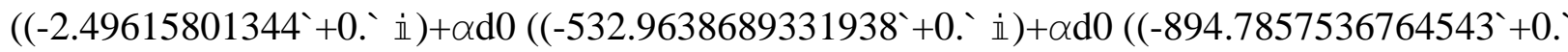

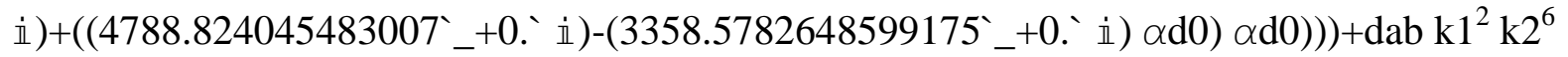

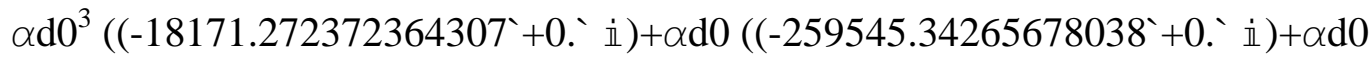

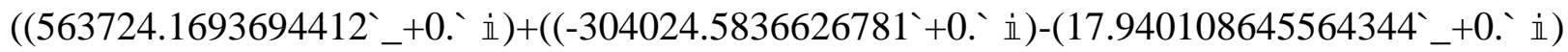

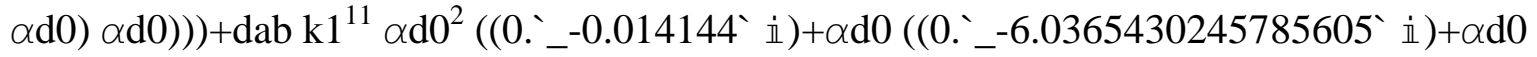

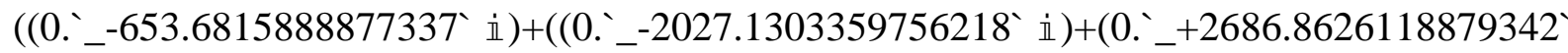

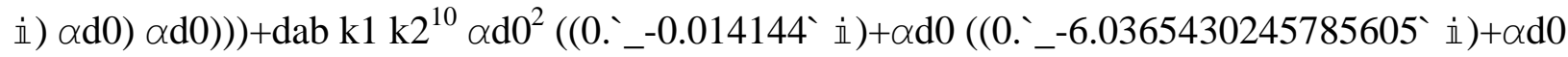

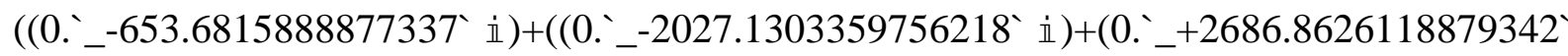

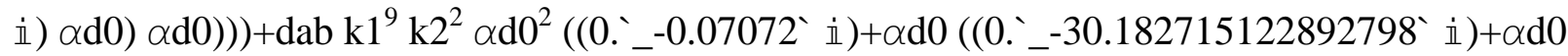

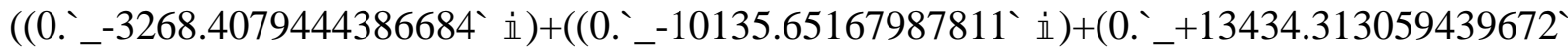

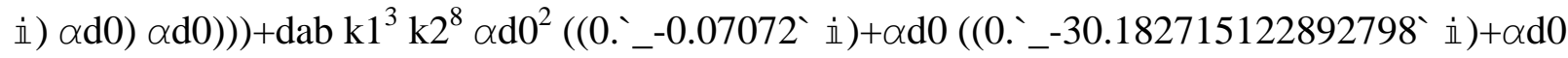

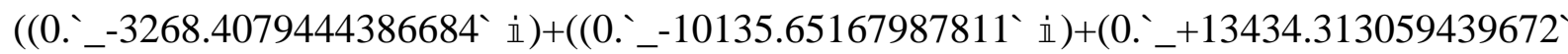

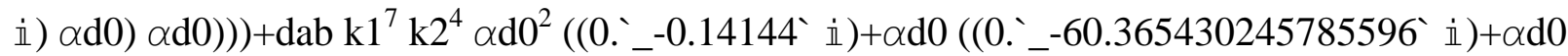

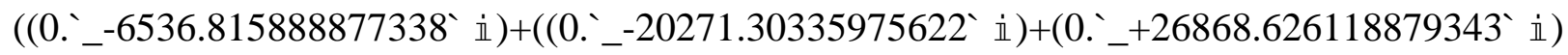

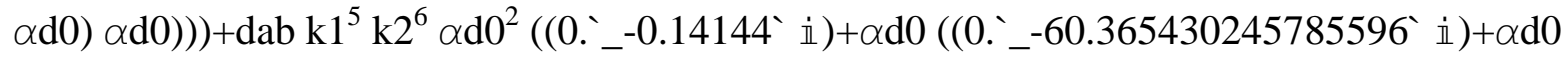

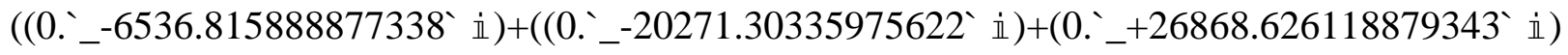

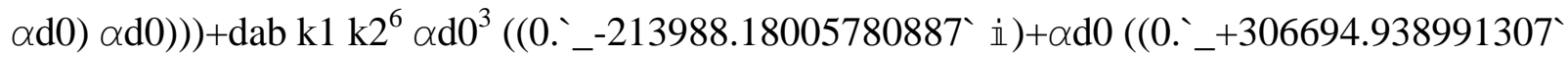

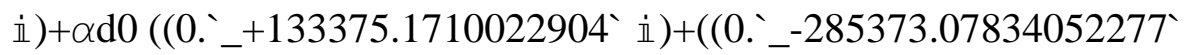

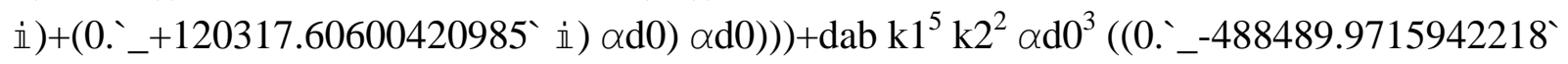

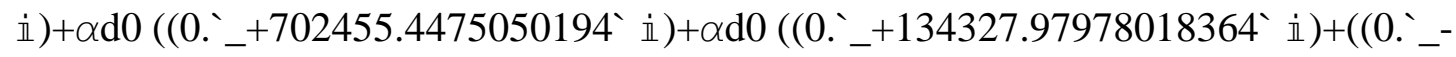

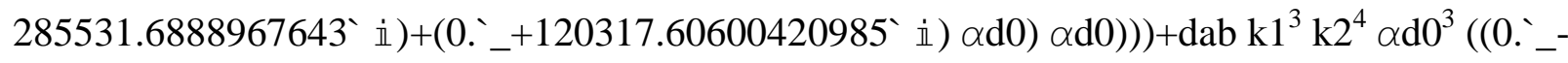
565227.2558838242 立 $)+\alpha \mathrm{d} 0\left(\left(0 ._{-}+811270.1322394701^{`} \dot{\mathbb{1}}\right)+\alpha \mathrm{d} 0\right.$

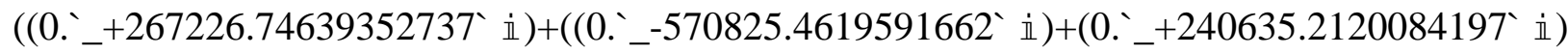
$\alpha \mathrm{d} 0) \alpha \mathrm{d} 0)))+\mathrm{s}^{5}\left(\mathrm{k}^{2} \alpha \mathrm{d} 0^{2}\left(0.24987951470397993^{\prime}+\alpha \mathrm{d} 0\left(-0.24895806744991003{ }^{`}+(-\right.\right.\right.$

$\left.\left.\left.0.24742873934734153+0.24773437872046827^{`} \alpha \mathrm{d} 0\right) \alpha \mathrm{d} 0\right)\right)+\mathrm{k} 2^{2} \alpha \mathrm{d} 0^{2}$ $\left(0.24987951470397993 \_+\alpha \mathrm{d} 0(-0.24895806744991003 `+(-\right.$ $\left.\left.\left.\left.0.24742873934734153^{`}+0.24773437872046827^{`} \alpha \mathrm{d} 0\right) \alpha \mathrm{d} 0\right)\right)\right)+\mathrm{dab} \mathrm{k} 1^{10} \alpha \mathrm{d} 0^{3}$

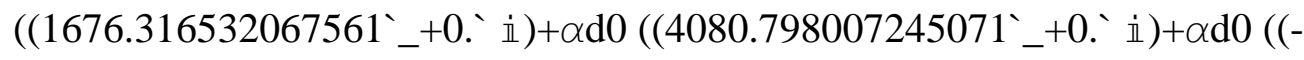

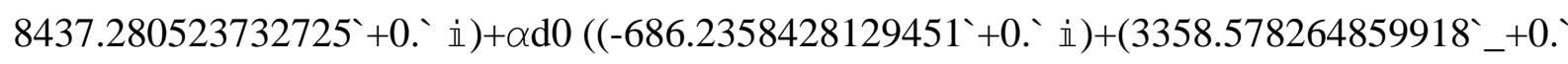

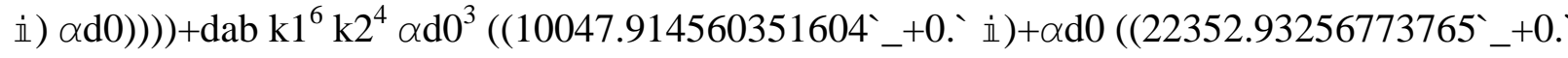
$\dot{\mathrm{i}})+\alpha \mathrm{d} 0\left(\left(-54202.826157102165+0\right.\right.$ ` $\left.^{\mathrm{i}}\right)+\alpha \mathrm{d} 0\left(\left(15037.881125054353{ }^{\prime}+0\right.\right.$.

i் $)+(6717.156529719836$ _+0.'立 $) \alpha \mathrm{d} 0))))+\mathrm{dab} \mathrm{k} 1^{8} \mathrm{k} 2^{2} \alpha \mathrm{d} 0^{3}\left(\left(6702.769970256803^{`}+0\right.\right.$.

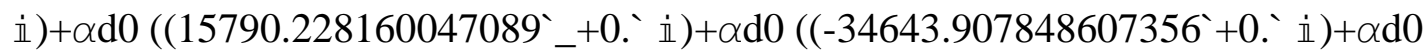

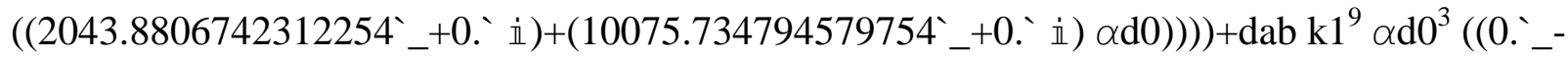

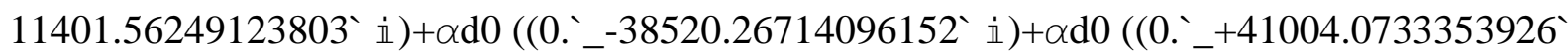




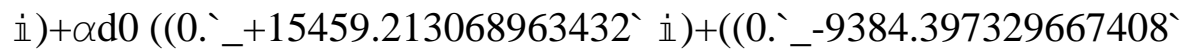

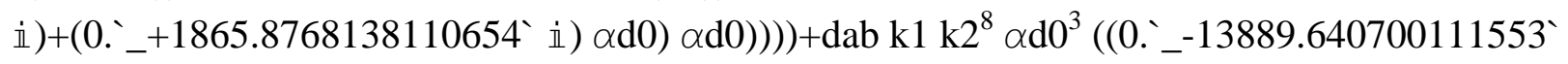

i) $+\alpha \mathrm{d} 0\left(\left(0 .{ }_{-}-34581.570639101104\right.\right.$ i $)+\alpha \mathrm{d} 0\left(\left(0 .{ }_{-}+50676.1217803853 ` \dot{1}\right)+\alpha \mathrm{d} 0\right.$

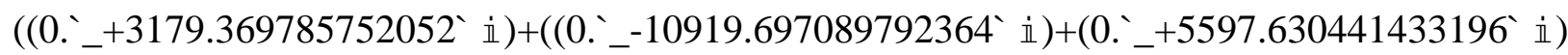
$\alpha \mathrm{d} 0) \alpha \mathrm{d} 0))))+\mathrm{dab} \mathrm{k} 1^{7} \mathrm{k}^{2} \alpha \mathrm{d} 0^{3}\left(\left(0 ._{-}-48094.328173825655^{\circ} \dot{\mathrm{i}}\right)+\alpha \mathrm{d} 0\left(\left(0 ._{-}^{\prime}-\right.\right.\right.$

150142.37206198566 i $)+\alpha \mathrm{d} 0\left(\left(0{ }^{\prime} \_+173688.34178656313 ` \dot{\mathrm{i}}\right)+\alpha \mathrm{d} 0\right.$

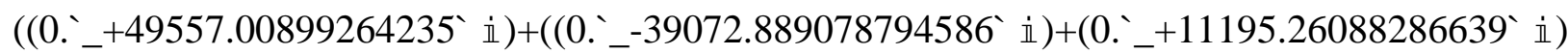
$\alpha \mathrm{d} 0) \alpha \mathrm{d} 0))))+\mathrm{dab} \mathrm{k} 1^{3} \mathrm{k}^{6} \alpha \mathrm{d} 0^{3}\left(\left(0 ._{-}-53070.484591572684\right.\right.$ i) $)+\alpha \mathrm{d} 0\left(\left(0 .{ }_{-}-\right.\right.$

$142264.9790582648 ` \dot{i})+\alpha \mathrm{d} 0\left(\left(0 .{ }_{-}+193032.43867654848 ` \dot{1}\right)+\alpha \mathrm{d} 0\right.$ $\left(\left(0 .{ }_{-}+24997.322426219594\right.\right.$ i $)+\left(\left(0 ._{-}-42143.488599044504\right.\right.$ i $)+\left(0 .^{\prime}+18658.768138110656\right.$

i) $\alpha \mathrm{d} 0) \alpha \mathrm{d} 0))))+\mathrm{dab} \mathrm{k1} 1^{5} \mathrm{k} 2^{4} \alpha \mathrm{d} 0^{3}\left(\left(0 .{ }_{-}-75873.60957404875 \circ\right.\right.$ i $)+\alpha \mathrm{d} 0\left(\left(0 ._{-}^{\prime}-\right.\right.$ 219305.51334018784 i $)+\alpha \mathrm{d} 0\left(\left(0 .{ }_{-}+275040.5853473337\right.\right.$ i $)+\alpha \mathrm{d} 0$

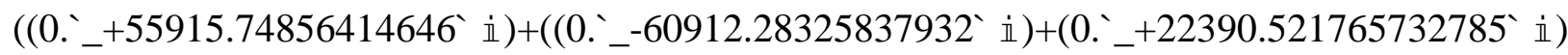
$\alpha \mathrm{d} 0) \alpha \mathrm{d} 0))))+\mathrm{dab} \mathrm{k} 1^{4} \mathrm{k}^{4} \alpha \mathrm{d} 0^{3}\left(\left(42755.90805494464 \_+0 . ` \dot{1}\right)+\alpha \mathrm{d} 0((-\right.$ $420589.71583524125+0$.`立 $)+\alpha \mathrm{d} 0\left(\left(588423.9633569273{ }^{\prime}+0 . ` \dot{1}\right)+\alpha \mathrm{d} 0((-\right.$ $265239.58292031055+0$.`立 $)+((-41.86025350631604$ ` +0 .

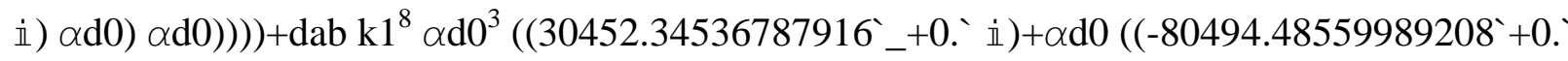

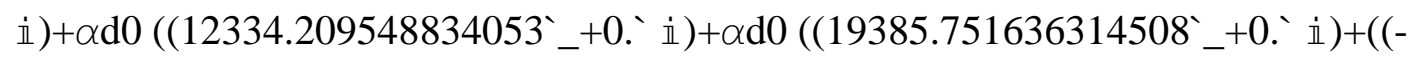
5.980036215187351 - +0 .

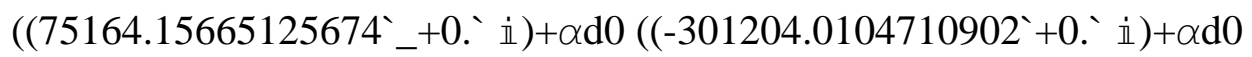
$\left(\left(220820.1985132498 \_+0 . ` i\right)+\alpha \mathrm{d} 0((-49637.191877561636+0 . ` \dot{1})+((-\right.$ $29.900181075939045+0 . ` \dot{1})+\left(1.6193107059010058^{\prime * \wedge}-12+0\right.$.

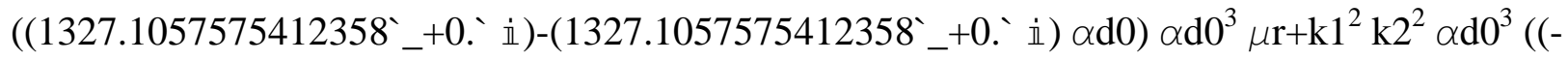
$1.0073532466900996 * \wedge 6+0$.`i $)+\left(\left(2.014790685058888^{* * \wedge} 6+0 . ` \dot{1}\right)-\right.$ $\left(1.0074374383687885^{* * \wedge} 6+0\right.$.`i $\left.\left.) \alpha \mathrm{d} 0\right) \alpha \mathrm{d} 0\right) \mu \mathrm{r}+\mathrm{k} 2^{4} \alpha \mathrm{d} 0^{3}\left(\left(-1.0086803524476408^{* * \wedge} 6+0\right.\right.$.

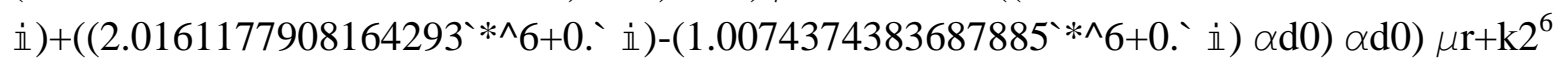

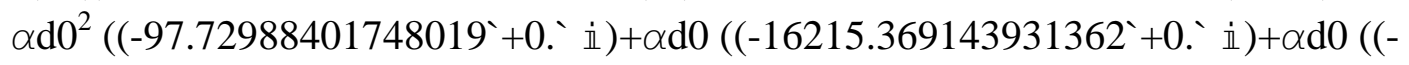
$59665.1516927372+0$.

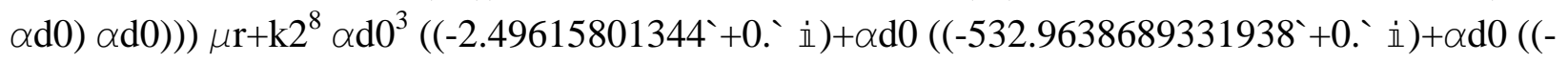

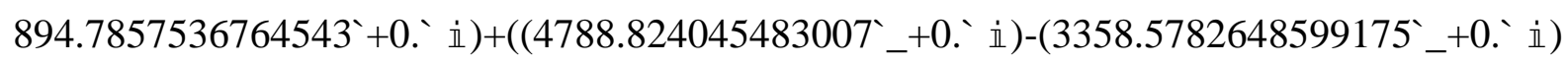

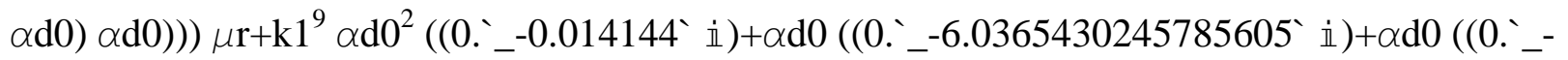
653.6815888877337 i $)+\left(\left(0 .{ }_{-}-2027.1303359756218\right.\right.$ i $\left.)+\left(0 .{ }_{-}+2686.8626118879342 ` \dot{i}\right) \alpha \mathrm{d} 0\right)$

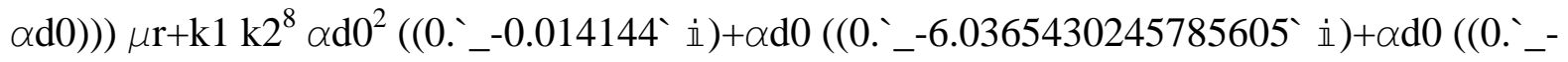

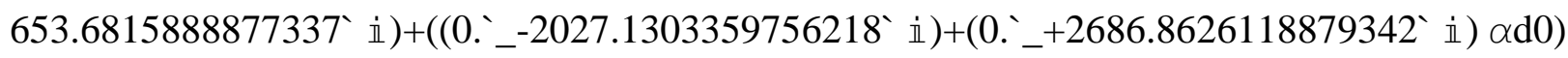

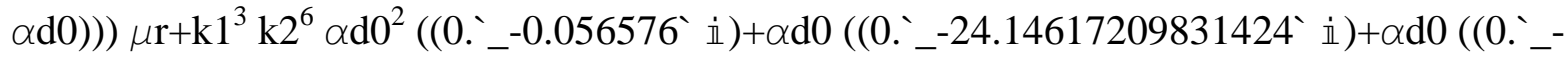
2614.726355550935 i $)+\left(\left(0 ._{-}-8108.521343902488^{`} \dot{\mathrm{i}}\right)+\left(0 ._{-}+10747.450447551737\right.\right.$ i $\left.) \alpha \mathrm{d} 0\right)$

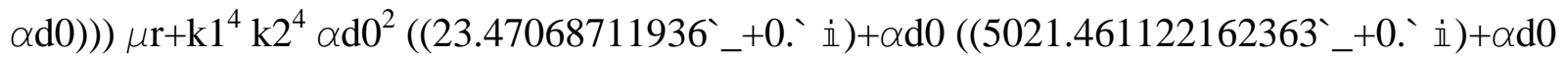
$\left(\left(10643.50241493563 `+0 . ` i \frac{1}{1}\right)+\alpha \mathrm{d} 0((-27996.198832227536+0 . `\right.$

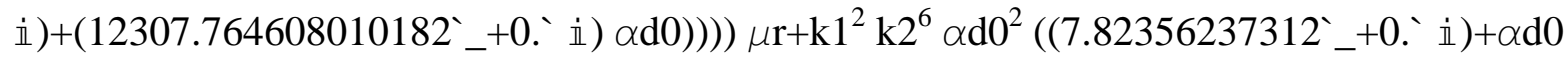
$\left(\left(1668.828058027241{ }^{\prime}+0 . ` \dot{1}\right)+\alpha \mathrm{d} 0\left(\left(2481.90640044549 \_+0 . ` i \frac{1}{)}\right)+\alpha \mathrm{d} 0((-\right.\right.$ 


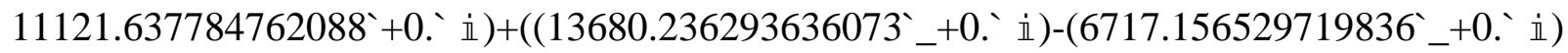
$\alpha \mathrm{d} 0) \alpha \mathrm{d} 0)))) \mu \mathrm{r}+\mathrm{k} 1^{2} \mathrm{k} 2^{4} \alpha \mathrm{d} 0^{2}\left(\left(18132.699315044607 \_+0 . ` \dot{1}\right)+\alpha \mathrm{d} 0((-\right.$

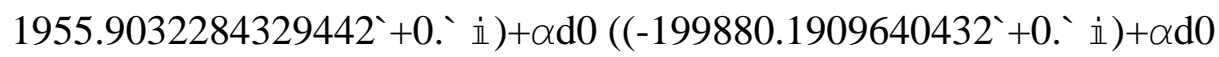

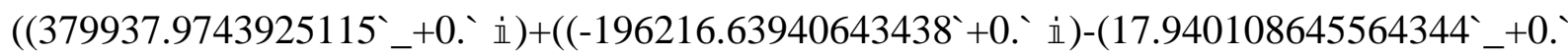
ii) $\alpha \mathrm{d} 0) \alpha \mathrm{d} 0)))) \mu \mathrm{r}+\mathrm{k} 1 \mathrm{k} 2^{4} \alpha \mathrm{d} 0^{2}\left(\left(0 ._{-}{ }_{-}-61026.45759947559{ }^{\circ} \dot{1}\right)+\alpha \mathrm{d} 0\left(\left(0 ._{-}^{\prime}-\right.\right.\right.$ 213988.18005780884 i $)+\alpha \mathrm{d} 0\left(\left(0 .^{\prime}+306694.93899130693 ` \dot{1}\right)+\alpha \mathrm{d} 0\right.$

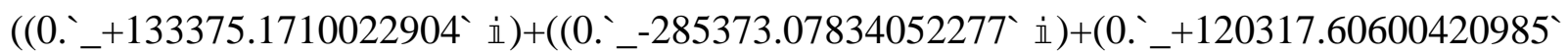
i) $\alpha \mathrm{d} 0) \alpha \mathrm{d} 0)))) \mu \mathrm{r}+\mathrm{k} 1^{3} \mathrm{k}^{2} \alpha \mathrm{d} 0^{2}\left(\left(0 .{ }_{-}-122052.91519895117\right.\right.$ i $)+\alpha \mathrm{d} 0\left(\left(0 ._{-}-\right.\right.$ 351239.0758260153 i $)+\alpha \mathrm{d} 0\left(\left(0 `_{-}+504575.1932481631\right.\right.$ i $)+\alpha \mathrm{d} 0\left(\left(0 .{ }_{-}+133851.575391237\right.\right.$

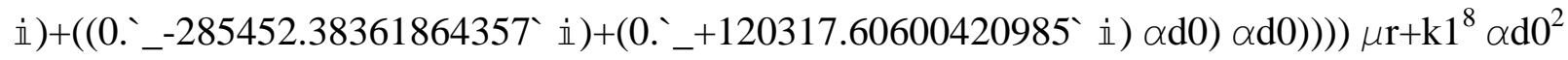
$\left(\left(7.82356237312 \_+0\right.\right.$ ○立 $)+\alpha \mathrm{d} 0\left(\left(1676.316532067561 \_+0 . ` \dot{1}\right)+\alpha \mathrm{d} 0\right.$ $\left(\left(4080.7980072450711_{-}+0 . ` i \frac{1}{)}+\alpha \mathrm{d} 0((-8437.280523732725+0 . ` i)+\alpha \mathrm{d} 0((-\right.\right.$

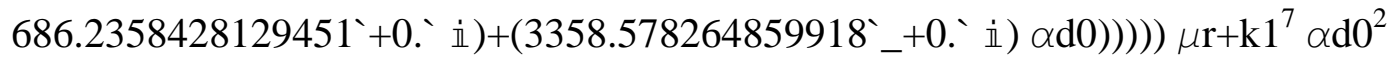
$\left(\left(0 ._{-}+977.063743699873\right.\right.$ i) $)+\mathrm{k} 2^{2}\left(\left(0 ._{-}-0.056576\right.\right.$ i $)+\alpha \mathrm{d} 0\left(\left(0 ._{-}{ }_{-}-24.14617209831424\right.\right.$ i $)+\alpha \mathrm{d} 0\left(\left(0 .{ }_{-}-2614.726355550935\right.\right.$ i $)+\left(\left(0 .^{\prime}-8108.5213439024888^{`}\right.\right.$ i் $)+\left(0 .{ }_{-}+10747.450447551737\right.$ i $\left.\left.\left.\left.) \alpha \mathrm{d} 0\right) \alpha \mathrm{d} 0\right)\right)\right)+\alpha \mathrm{d} 0\left(\left(0 .{ }_{-}-11401.56249123803 ` \dot{1}\right)+\alpha \mathrm{d} 0\right.$ $\left(\left(0 ._{-}-38520.26714096152\right.\right.$ i $)+\alpha \mathrm{d} 0\left(\left(0 ._{-}+41004.0733353926\right.\right.$ i $)+\alpha \mathrm{d} 0$ $\left(\left(0 .{ }_{-}+15459.213068963432 ` \dot{i}\right)+\left(\left(0{ }^{\prime}{ }_{-}-9384.397329667408 ` \dot{i}\right)+\left(0 .{ }^{\prime}+1865.8768138110654\right.\right.\right.$

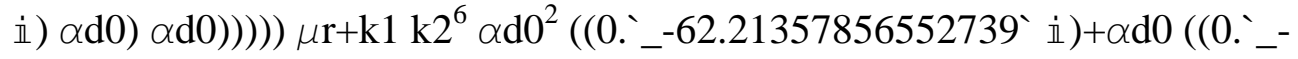

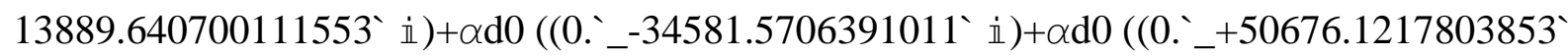
ii $)+\alpha \mathrm{d} 0\left(\left(0 ._{-}+3179.369785752052 ` \dot{1}\right)+\left(\left(0 ._{-}-10919.697089792364\right.\right.\right.$ i $)+\left(0 ._{-}+5597.630441433196\right.$ i $\left.\left.\left.\left.\left.\left.) \alpha \mathrm{d} 0\right) \alpha \mathrm{d} 0\right)\right)\right)\right)\right) \mu \mathrm{r}+\mathrm{k}^{3}{ }^{3} \mathrm{k}^{4} \alpha \mathrm{d} 0^{2}\left(\left(0 ._{-}+852.6365865688183\right.\right.$ i) $+\alpha \mathrm{d} 0\left(\left(0 ._{-}-39180.84389146113 ` i \frac{1}{1}\right)+\alpha \mathrm{d} 0\left(\left(0 ._{-}-107683.40841916372 ` \dot{1}\right)+\alpha \mathrm{d} 0\right.\right.$

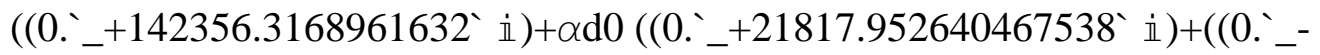
31223.791509252136 i

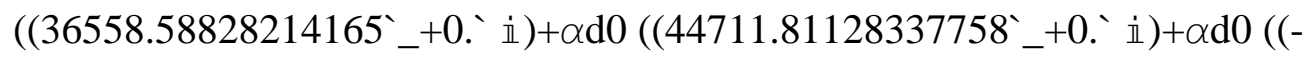

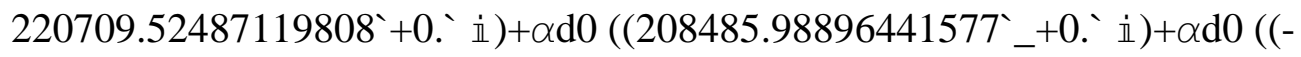

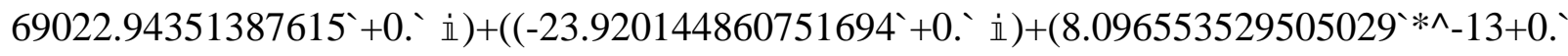
i) $\alpha \mathrm{d} 0) \alpha \mathrm{d} 0))))) \mu \mathrm{r}+\mathrm{k} 1^{6} \alpha \mathrm{d} 0^{2}\left(\left(18328.159083079565_{-}^{\prime}+0 .^{`} \dot{1}\right)+\alpha \mathrm{d} 0\right.$ $\left(\left(30452.34536787916 \_+0 . ` \dot{1}\right)+\alpha \mathrm{d} 0((-80494.48559989208 `+0 ` \grave{1})+\alpha \mathrm{d} 0\right.$ $\left(\left(12334.209548834055 \_+0 . \grave{i}\right)+\alpha \mathrm{d} 0\left(\left(19385.751636314508 \_+0 . ` i\right)+((-\right.\right.$

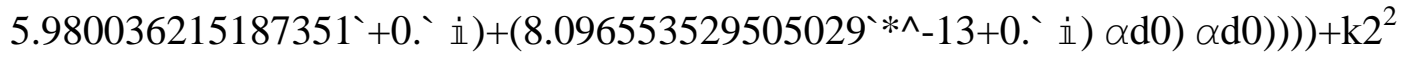

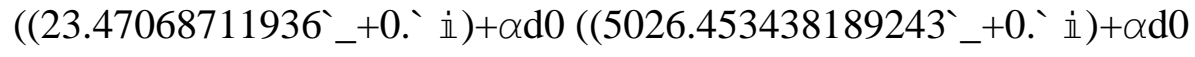

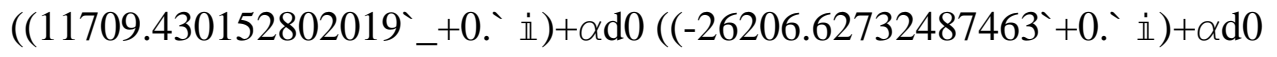

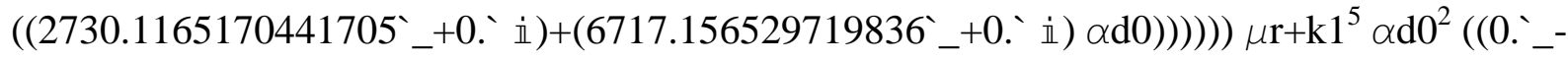
61026.45759947559 i $)+\alpha \mathrm{d} 0\left(\left(0 .^{\prime}-137250.89576820648 ` \dot{i}\right)+\alpha \mathrm{d} 0\right.$

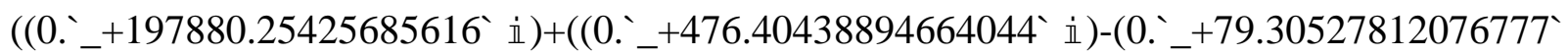

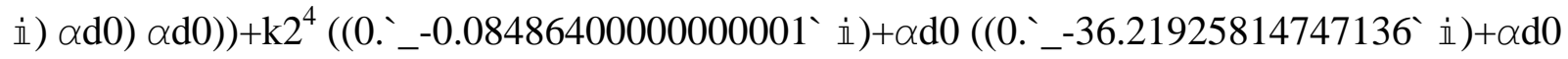

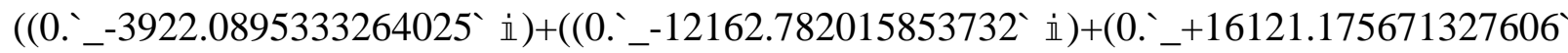
ii) $\alpha \mathrm{d} 0) \alpha \mathrm{d} 0)))+\mathrm{k} 2^{2}\left(\left(0 .^{\prime}+1891.9139088342188^{`} \dot{\mathrm{i}}\right)+\alpha \mathrm{d} 0\left(\left(0 .{ }_{-}-36692.76568258761\right.\right.\right.$ i $)+\alpha \mathrm{d} 0$ 


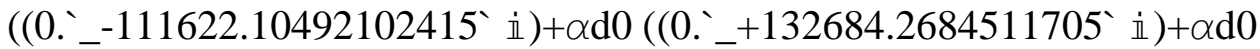

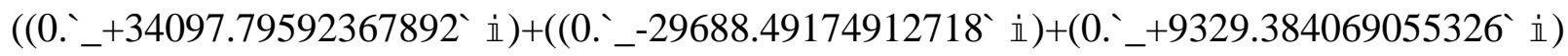
$\alpha \mathrm{d} 0) \alpha \mathrm{d} 0)))))) \mu \mathrm{r}+\mathrm{s}^{4}\left(\left(58.372291581682816_{-}{ }_{-} 1.0587911840678754 * 4^{*}-22\right.\right.$ i $) \mathrm{k} 1^{2}$

$\alpha \mathrm{d} 0^{2}+\left(58.372291581682816^{\prime}-1.0587911840678754^{` * \wedge}-22 \dot{\mathrm{i}}\right) \mathrm{k} 2^{2} \alpha \mathrm{d} 0^{2}-58.228702042800414$ $\mathrm{k} 1^{2} \alpha \mathrm{d} 0^{3}-58.228702042800414{ }^{`} 2^{2} \alpha \mathrm{d} 0^{3}+\mathrm{dab} \mathrm{k} 1^{2} \mathrm{k} 2^{2} \alpha \mathrm{d} 0^{2}((-0.4997590294079599+0$ ``

i) $+\alpha \mathrm{d} 0\left(\left(0.4979161348998199{ }^{\prime}+0\right.\right.$.'立 $)+((0.49485747869468305$ _+0.' $\dot{\mathbb{1}})-$ $(0.49546875744093655$ _+0.'立 $) \alpha \mathrm{d} 0) \alpha \mathrm{d} 0))+\mathrm{dab} \mathrm{k} 1^{4} \alpha \mathrm{d} 0^{2}((-0.24987951470397995$ + +0 .

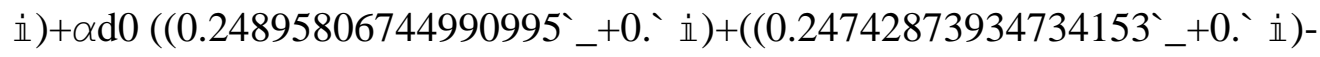

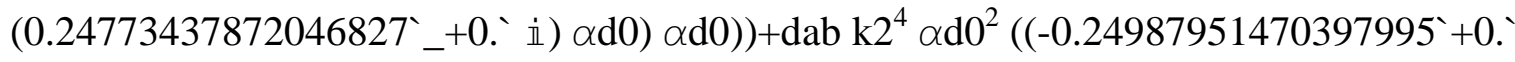

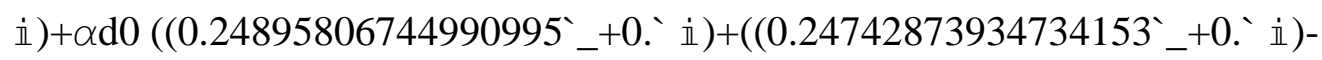

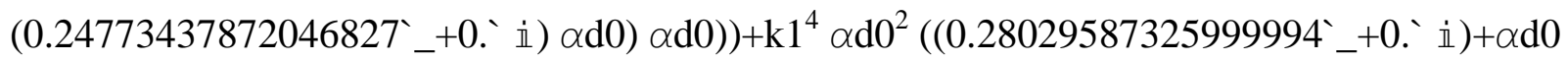
$\left(\left(8.110687720789617 \_+0 ` \dot{1}\right)+\alpha \mathrm{d} 0((-8.501242690333886+0\right.$ ‘

i் $)+(4.375037960926727$ _+0`立 $) \alpha \mathrm{d} 0)))+\mathrm{k} 2^{4} \alpha \mathrm{d} 0^{2}\left(\left(0.28029587325999994{ }_{-}+0\right.\right.$. $((8.110687720789617+0 . ` \dot{1})+\alpha \mathrm{d} 0((-8.501242690333886+0$ ‘

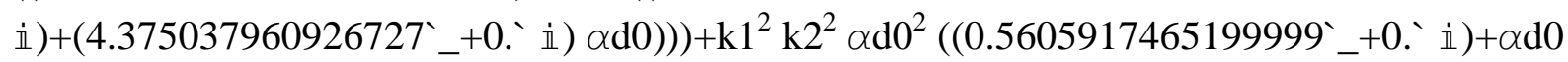
$\left(\left(16.221375441579234 \_+0\right.\right.$. $\left.\dot{\mathrm{i}}\right)+\alpha \mathrm{d} 0\left(\left(-17.00248538066777+0\right.\right.$.` $^{`}$

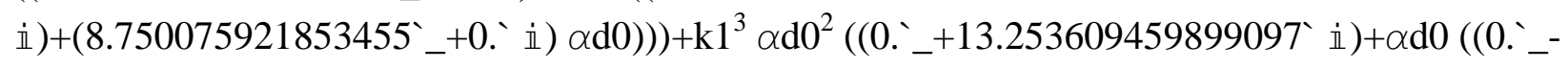

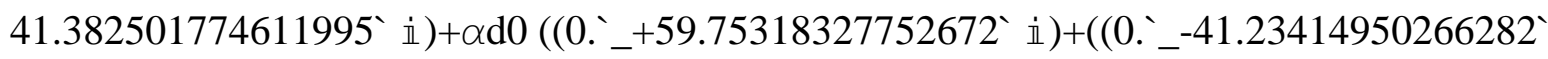

i) $\left.\left.\left.\left.)+\left(0 .{ }_{-}+9.631553431417828^{`} \dot{\mathbb{i}}\right) \alpha \mathrm{d} 0\right) \alpha \mathrm{d} 0\right)\right)\right)+\mathrm{k} 1 \mathrm{k} 2^{2} \alpha \mathrm{d} 0^{2}\left(\left(0 .{ }_{-}+13.253609459899097\right.\right.$

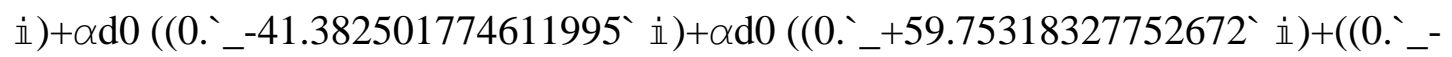

41.23414950266282 ì $)+\left(0 .^{\prime}+9.631553431417828\right.$ ì $\left.\left.\left.\left.) \alpha \mathrm{d} 0\right) \alpha \mathrm{d} 0\right)\right)\right)+\mathrm{k} 1^{2} \alpha \mathrm{d} 0^{2}((-$

$0.24987951470397995+0$ ` $\left.^{\circ}\right)+\alpha \mathrm{d} 0\left(\left(0.24895806744990995 \_+0\right.\right.$.

i் $)+((0.24742873934734153$ _+0.

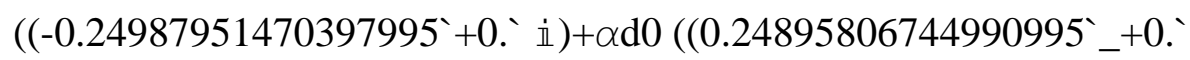

i) $+((0.24742873934734153$ _+0.

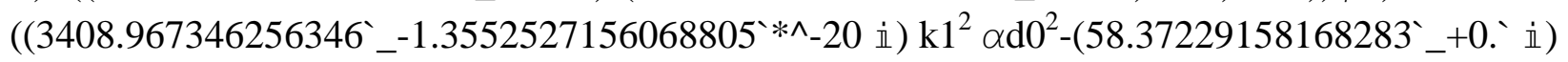
dab k1 $1^{4} \alpha \mathrm{d}^{2}+\left(3408.967346256346_{-}^{\prime}-1.3552527156068805^{` *}{ }^{\prime}-20\right.$ i் $) \mathrm{k} 2^{2} \alpha \mathrm{d} 0^{2}-$

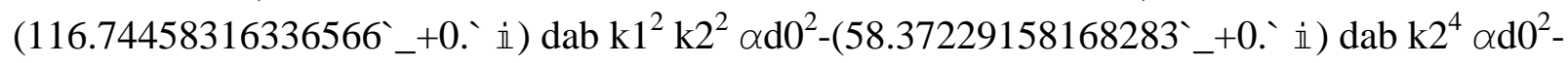
$3404.7667553568335 \mathrm{k}^{2} \alpha \mathrm{d} 0^{3}+\left(58.22870204280039^{\prime}+0\right.$.`i $\left.^{\prime}\right) \mathrm{dab} \mathrm{k} 1^{4} \alpha \mathrm{d} 0^{3}$ -

$3404.7667553568335 \mathrm{k}^{2} \alpha \mathrm{d} 0^{3}+\left(116.45740408560079^{`}+0\right.$ `` $\left.^{2}\right) \mathrm{dab} \mathrm{k}^{2} \mathrm{k} 2^{2}$ $\alpha \mathrm{d} 0^{3}+\left(58.22870204280039{ }_{-}+0\right.$ `' $\left.^{\mathrm{i}}\right) \mathrm{dab} \mathrm{k} 2^{4} \alpha \mathrm{d} 0^{3}+\mathrm{k} 1^{4} \mathrm{k} 2^{2} \alpha \mathrm{d} 0^{2}\left(\left(0.09245507999999998^{\prime}+0\right.\right.$.

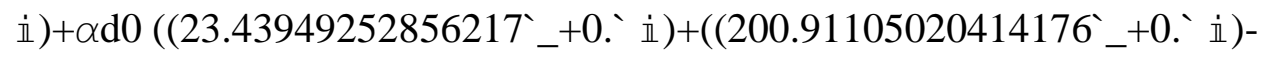

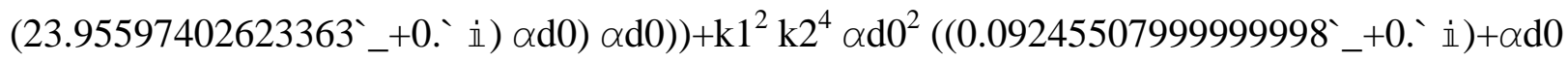

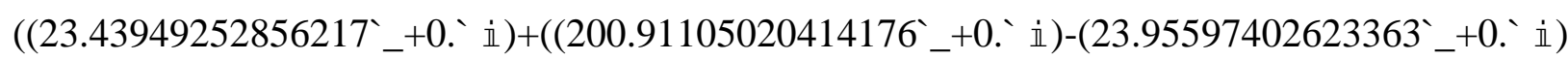

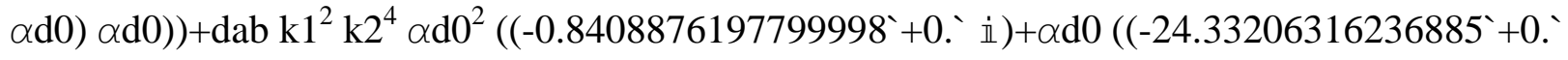
$\dot{\mathbb{1}})+\left(\left(25.503728071001657 \_+0\right.\right.$. $\left.\dot{\mathbb{1}}\right)-\left(13.125113882780182^{\prime}+0\right.$. $\left.\left.\left.\left.\dot{\mathbb{1}}\right) \alpha \mathrm{d} 0\right) \alpha \mathrm{d} 0\right)\right)+\mathrm{dab} \mathrm{k} 1^{4} \mathrm{k} 2^{2}$

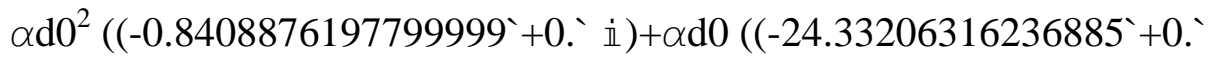

$\left.\left.\dot{\mathbb{1}})+\left(\left(25.50372807100166{ }_{-}+0{ }^{`} \dot{\mathbb{1}}\right)-\left(13.125113882780182_{-}{ }^{\prime}+0{ }^{`} \dot{\mathbb{1}}\right) \alpha \mathrm{d} 0\right) \alpha \mathrm{d} 0\right)\right)+\mathrm{k} 1^{6} \alpha \mathrm{d} 0^{2}$ $\left(\left(0.030818359999999996 \_+0 . \dot{1}\right)+\alpha \mathrm{d} 0\left(\left(7.8131641761873905{ }^{\prime}+0\right.\right.\right.$.

i் $)+\left(\left(66.97035006804725^{\prime}+0\right.\right.$. 


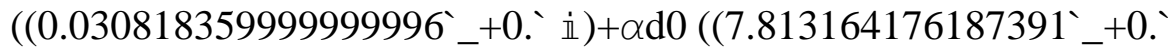

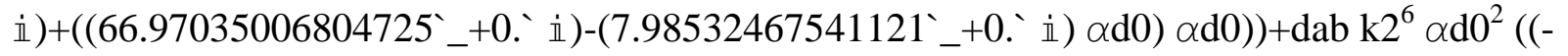
$0.28029587325999994+0$ ‘ $\left.^{-1}\right)+\alpha \mathrm{d} 0\left(\left(-8.110687720789617{ }^{`}+0\right.\right.$.

i) $+\left(\left(8.501242690333886{ }_{-}+0\right.\right.$.

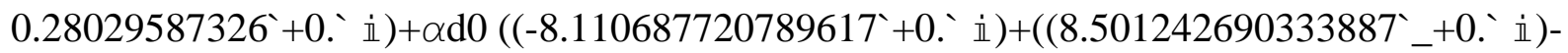

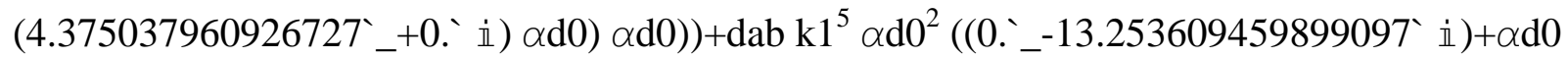
$\left(\left(0\right.\right.$ ___$^{\prime}+41.382501774611995$ i $)+\alpha \mathrm{d} 0\left(\left(0\right.\right.$. $_{-}-59.753183277526722^{\prime}$

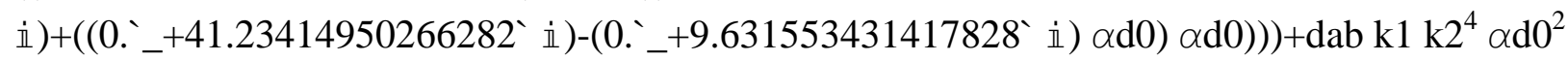
$\left(\left(0 .{ }_{-}-13.253609459899097\right.\right.$ ii $)+\alpha \mathrm{d} 0\left(\left(0\right.\right.$.__$_{-}+41.382501774611995$ i $)+\alpha \mathrm{d} 0\left(\left(0\right.\right.$. $_{-}-$

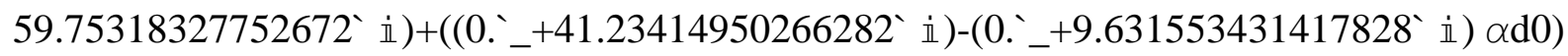

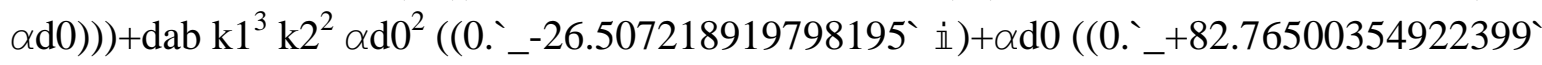

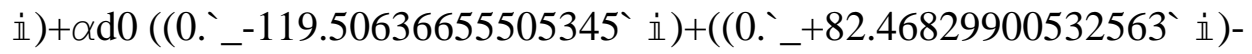

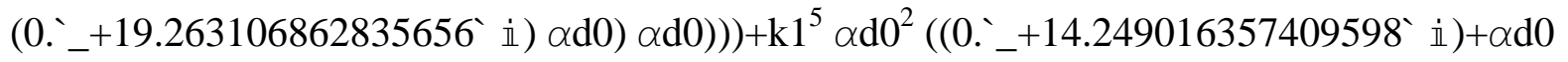

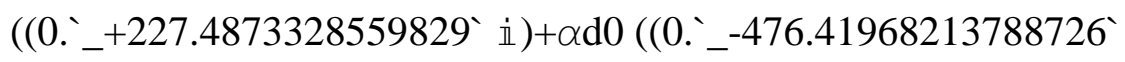

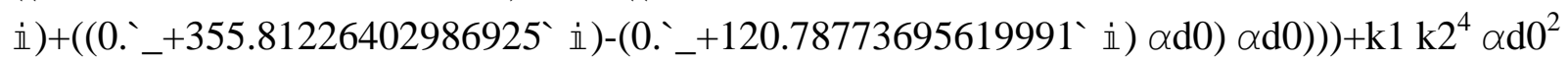
$\left(\left(4.741411591966076^{* \wedge}-15+14.249016357409598^{`} \dot{i}\right)+\alpha \mathrm{d} 0\left(\left(0\right.\right.\right.$ `__$^{*}+227.4873328559829^{\prime}$

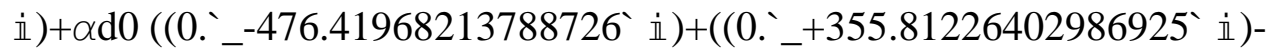
$\left(0 .{ }_{-}+120.78773695619991\right.$ i $\left.\left.\left.\left.) \alpha \mathrm{d} 0\right) \alpha \mathrm{d} 0\right)\right)\right)+\mathrm{k} 1^{3} \mathrm{k} 2^{2} \alpha \mathrm{d} 0^{2}\left(\left(6.7053685781514305 * 5^{\prime}\right.\right.$

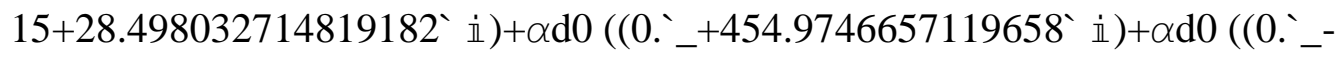
952.8393642757745 i் $)+\left(\left(0 .{ }_{-}+711.6245280597385\right.\right.$ i் $)-\left(0 .{ }_{-}+241.57547391239981\right.$ i $\left.) \alpha \mathrm{d} 0\right)$

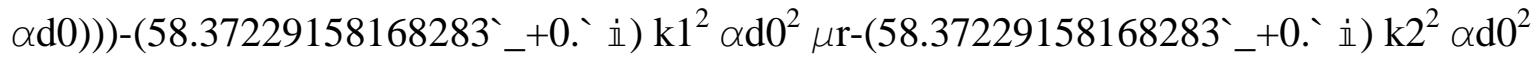

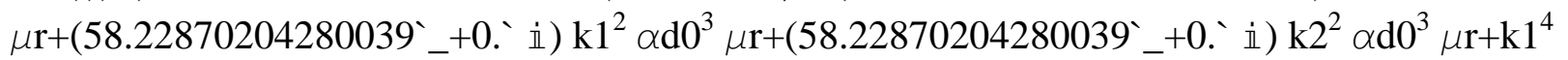
$\alpha \mathrm{d} 0^{2}\left(\left(-152.91514338058232 `+0{ }^{`} \dot{\mathbb{i}}\right)-\left(0.28029587325999994{ }_{-}+0 ` \dot{i}\right) \mu \mathrm{r}+\alpha \mathrm{d} 0\right.$

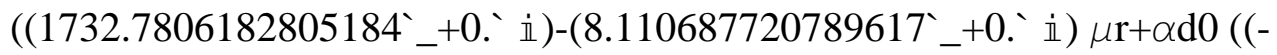

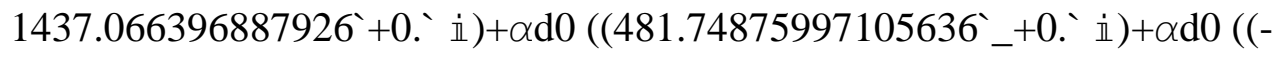
$157.63997123695725+0$. $\dot{\mathbb{1}})+(31.013177660695742$ _+0.`立 $\alpha \mathrm{d} 0)-(4.375037960926727$ _+0. i) $\left.\left.\left.\mu \mathrm{r})+\left(8.501242690333886{ }_{-}+0{ }^{`} \dot{\mathbb{1}}\right) \mu \mathrm{r}\right)\right)\right)+\mathrm{k} 2^{4} \alpha \mathrm{d} 0^{2}\left(\left(61.97757865056198_{-}+0 . ` \dot{1}\right)-\right.$

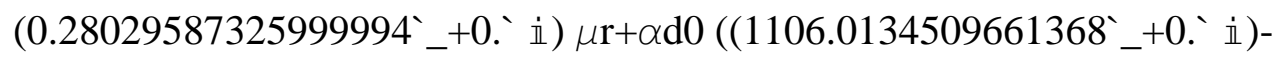

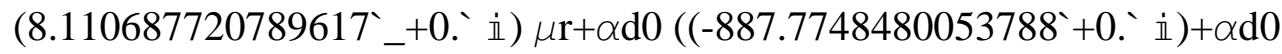
$\left(\left(466.0398569015887 \_+0\right.\right.$ ‘ $\left.\dot{\mathbb{1}}\right)+\alpha \mathrm{d} 0\left(\left(-341.37452712454547+0\right.\right.$ ` $^{`}$

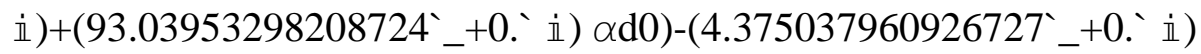
$\mu \mathrm{r})+\left(8.501242690333886{ }^{\prime}+0\right.$.'立 $\left.\left.\left.) \mu \mathrm{r}\right)\right)\right)+\mathrm{k} 1^{2} \mathrm{k} 2^{2} \alpha \mathrm{d} 0^{2}((-90.93756473002034+0$.`立 $)-$ $\left(0.5605917465199999{ }^{\prime}+0 . ` \dot{1}\right) \mu \mathrm{r}+\alpha \mathrm{d} 0\left(\left(2838.7940692466555_{-}+0\right.\right.$. $\left.\dot{1}\right)-$

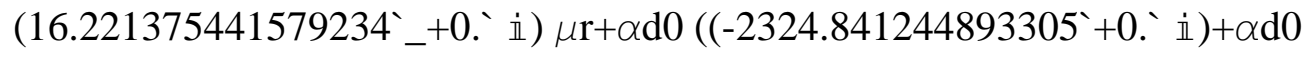
$\left(\left(947.788616872645 \_+0\right.\right.$ ` $\left.^{\prime} \dot{1}\right)+\alpha \mathrm{d} 0((-499.0144983615027+0$ `

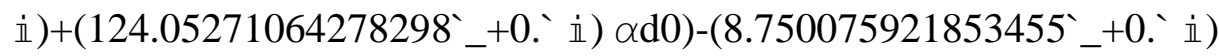

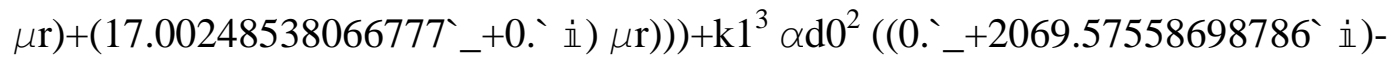

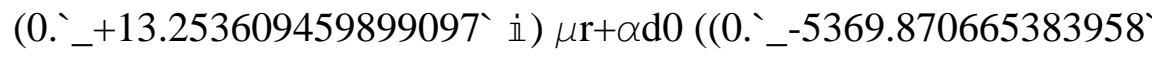

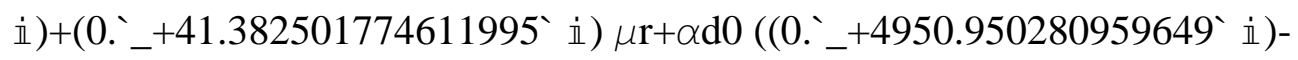

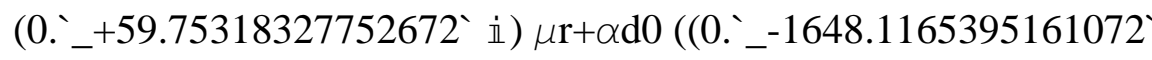




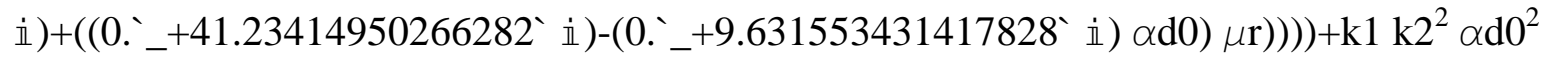
$\left(\left(0 .{ }_{-}+2069.57558698786\right.\right.$ i $)-\left(0 .{ }_{-}+13.253609459899097\right.$ i $) \mu \mathrm{r}+\alpha \mathrm{d} 0\left(\left(0 ._{-}{ }^{-}\right.\right.$ 5369.870665383958 i $)+\left(0 .{ }_{-}+41.382501774611995\right.$ i $) \mu \mathrm{r}+\alpha \mathrm{d} 0\left(\left(0 .{ }_{-}+4950.950280959649 `\right.\right.$

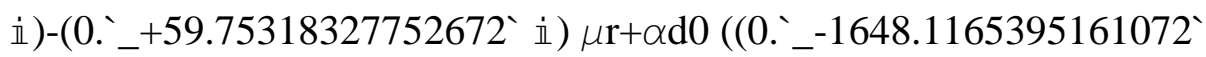
ii $)+\left(\left(0 .{ }^{\prime}+41.23414950266282\right.\right.$ i $\left.\left.\left.\left.\left.\left.)-\left(0 .^{\prime}+9.631553431417828^{`} \dot{i}\right) \alpha \mathrm{d} 0\right) \mu \mathrm{r}\right)\right)\right)\right)\right)+\mathrm{s}^{2}((-$

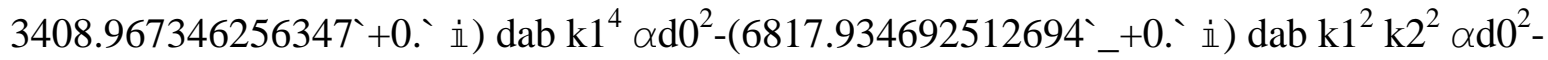
(3408.967346256347_+0.`立) dab k2 ${ }^{4} \alpha \mathrm{d} 0^{2}+\left(3404.7667553568326\right.$ _+0.'立) dab k1 ${ }^{4}$ $\alpha \mathrm{d} 0^{3}+(6809.533510713665$ _+0.`立 $) \mathrm{dab} \mathrm{k}^{2} \mathrm{k}^{2} \alpha \mathrm{d} 0^{3}+(3404.7667553568326$ _+0.`立 $) \mathrm{dab} \mathrm{k} 2^{4}$

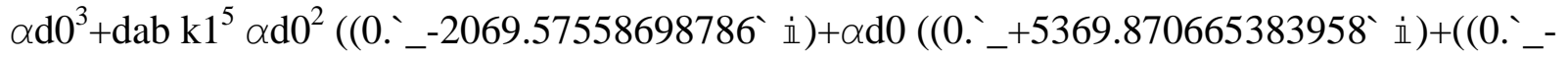

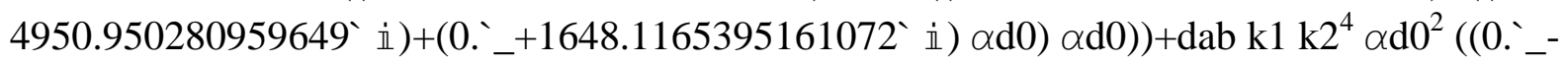
2069.57558698786 i) $)+\alpha \mathrm{d} 0\left(\left(0 .{ }^{\prime}+5369.870665383958\right.\right.$ i $)+\left(\left(0 .{ }^{\prime}-4950.950280959649{ }^{\prime}\right.\right.$ i) $)+\left(0 .{ }_{-}+1648.1165395161072\right.$ i $\left.\left.\left.) \alpha \mathrm{d} 0\right) \alpha \mathrm{d} 0\right)\right)+\mathrm{dab} \mathrm{k} 1^{3} \mathrm{k}^{2} \alpha \mathrm{d} 0^{2}\left(\left(0 ._{-}-4139.15117397572\right.\right.$

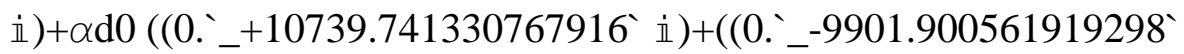

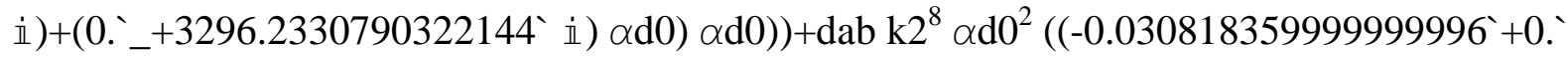
i $)+\alpha \mathrm{d} 0((-7.8131641761873905+0$. $\dot{\mathrm{i}})+\alpha \mathrm{d} 0((-66.97035006804725+0$.'

i $)+\left(7.98532467541121^{`}+0\right.$.

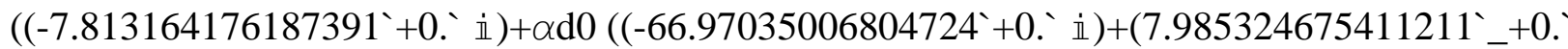
i) $\alpha \mathrm{d} 0)))+\mathrm{dab} \mathrm{k} 1^{6} \mathrm{k} 2^{2} \alpha \mathrm{d} 0^{2}\left(\left(-0.12327343999999998^{\circ}+0 `^{\prime} \dot{1}\right)+\alpha \mathrm{d} 0\left(\left(-31.252656704749562 `+0{ }^{`}\right.\right.\right.$

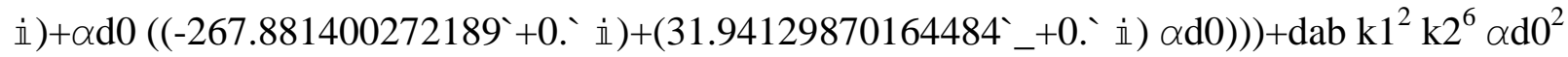
$\left((-0.12327343999999998 `+0\right.$. $\dot{1})+\alpha \mathrm{d} 0\left(\left(-31.252656704749562 `+0{ }^{`} \dot{1}\right)+\alpha \mathrm{d} 0((-\right.$

267.881400272189 +0.`立 $)+\left(31.94129870164484 \_+0\right.$.

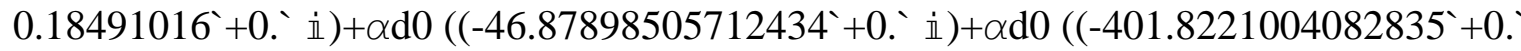

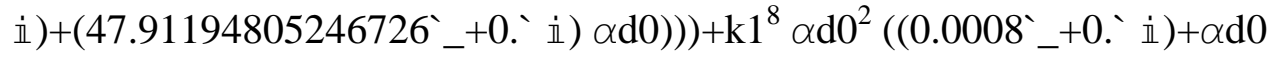
$\left(\left(0.38143342899200006 \_+00^{`} \dot{\mathrm{i}}\right)+\alpha \mathrm{d} 0\left(\left(45.78291000714875 \_+0\right.\right.\right.$.

ii $\left.\left.\left.)+\left(187.13424266650267 \_+0 . ` \dot{1}\right) \alpha \mathrm{d} 0\right)\right)\right)+\mathrm{k} 2^{8} \alpha \mathrm{d} 0^{2}\left(\left(0.0008_{-}+0 . ` \dot{\mathrm{i}}\right)+\alpha \mathrm{d} 0\right.$ $\left(\left(0.38143342899200006 \_+00^{`} \dot{1}\right)+\alpha \mathrm{d} 0\left(\left(45.78291000714875 \_+0 .\right.\right.\right.$

ii $)+\left(187.13424266650267 \_+0 .\right.$ i $\left.\left.\left.) \alpha \mathrm{d} 0\right)\right)\right)+\mathrm{k} 1^{2} \mathrm{k}^{6} \alpha \mathrm{dd} 0^{2}\left(\left(0.0031999999999999997^{\circ}+0\right.\right.$.

i $)+\alpha \mathrm{d} 0\left(\left(1.5257337159680002^{\prime}+0 . ` i \frac{1}{)}\right)+\alpha \mathrm{d} 0\left(\left(183.131640028595{ }^{\prime}+0\right.\right.\right.$.

i் $)+\left(748.5369706660108^{`}+0\right.$.

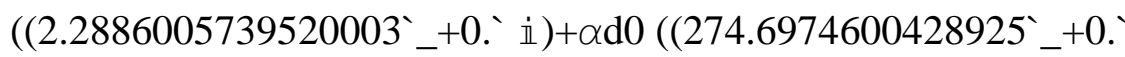

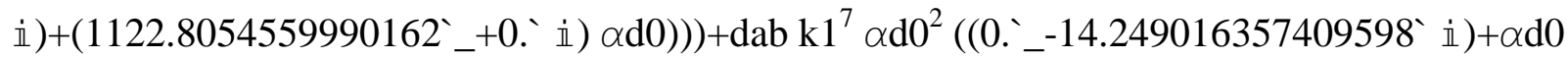
$\left(\left(0 .^{\prime}-227.4873328559829\right.\right.$ i $\left.\dot{1}\right)+\alpha \mathrm{d} 0\left(\left(0 .{ }_{-}+476.41968213788726\right.\right.$ i $)+\left(\left(0 .^{\prime}-\right.\right.$

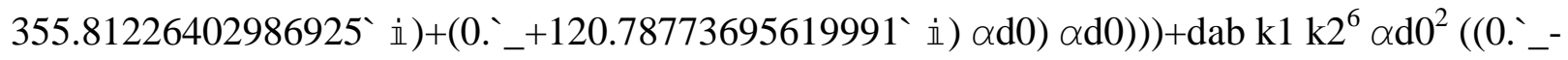
$\left.14.249016357409598^{`} \dot{i}\right)+\alpha \mathrm{d} 0\left(\left(0 .^{\prime}-227.4873328559829^{\circ} \dot{i}\right)+\alpha \mathrm{d} 0\right.$

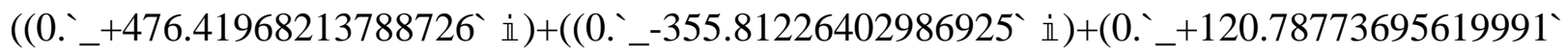

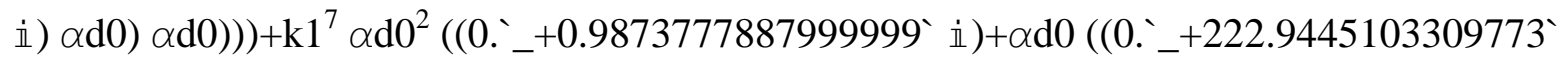

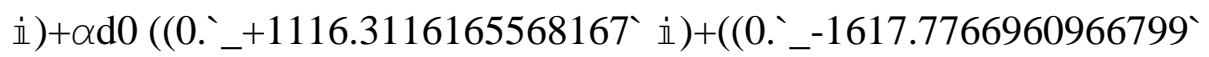

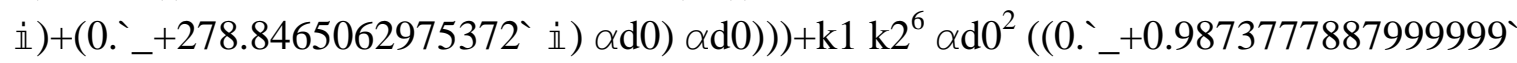
i $)+\alpha \mathrm{d} 0\left(\left(0 .{ }_{-}+222.9445103309773\right.\right.$ i $)+\alpha \mathrm{d} 0\left(\left(0 .{ }_{-}+1116.3116165568167\right.\right.$ i $)+\left(\left(0 ._{-}-\right.\right.$ 1617.7766960966806 i $)+\left(0 ._{-}+278.8465062975373\right.$ i $\left.\left.\left.\left.) \alpha \mathrm{d} 0\right) \alpha \mathrm{d} 0\right)\right)\right)+\mathrm{dab} \mathrm{k}^{5} \mathrm{k}^{2} \alpha \mathrm{d} 0^{2}\left(\left(0 ._{-}-\right.\right.$ 


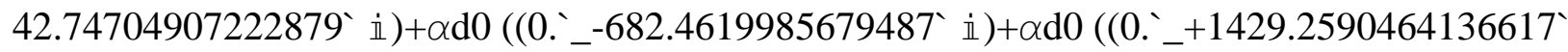

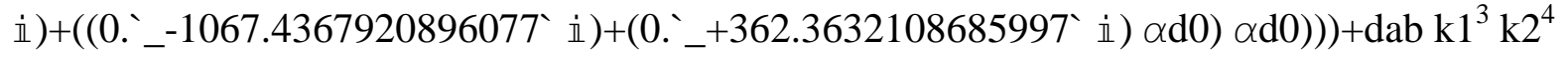

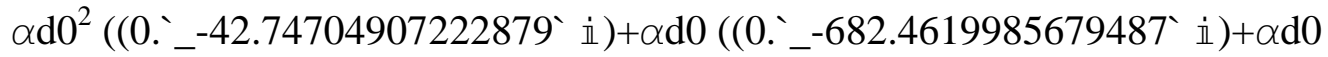

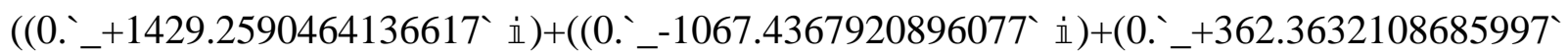

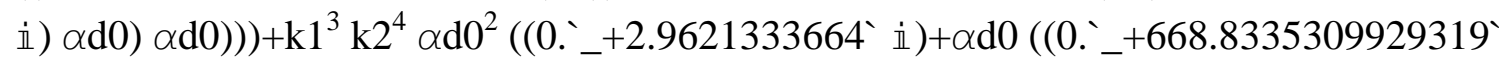

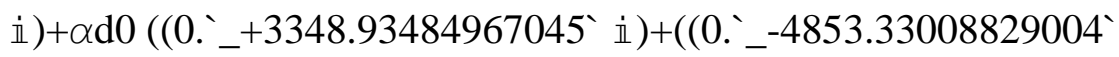

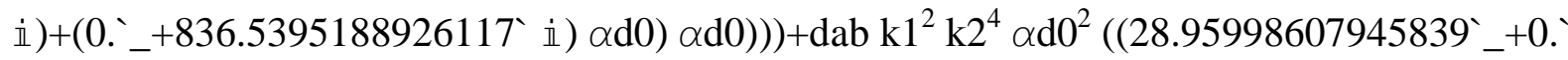

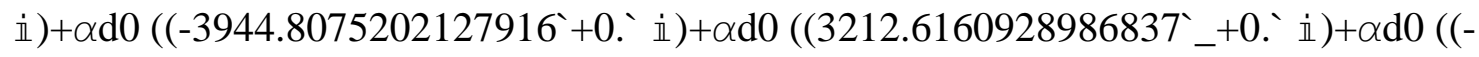

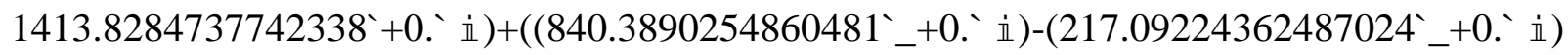
$\alpha \mathrm{d} 0) \alpha \mathrm{d} 0))))+\mathrm{dab} \mathrm{k} 1^{4} \mathrm{k} 2^{2} \alpha \mathrm{d} 0^{2}\left(\left(243.85270811060272 \_+0{ }^{`} \dot{i}\right)+\alpha \mathrm{d} 0((-\right.$ $4571.574687527173{ }^{`}+0$. $\left.\dot{\mathbf{1}}\right)+\alpha \mathrm{d} 0\left(\left(3761.907641781231^{\prime}+0\right.\right.$.` $\left.\dot{\mathbb{1}}\right)+\alpha \mathrm{d} 0((-$

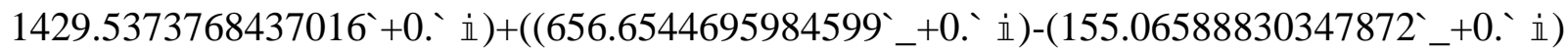

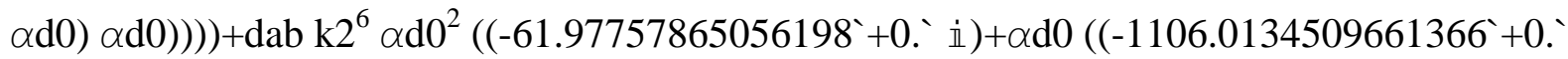

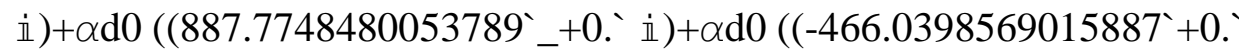

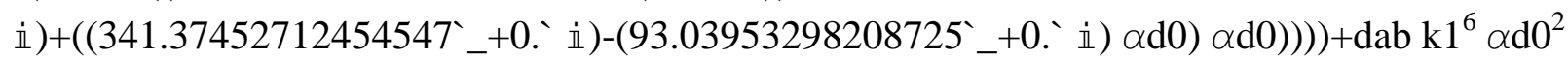

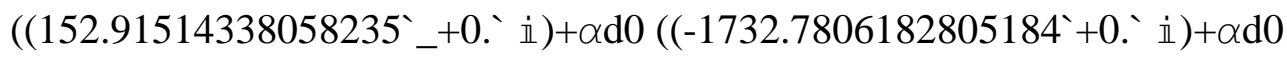
$\left(\left(1437.0663968879262 \_+0\right.\right.$.'立 $)+\alpha \mathrm{d} 0\left(\left(-481.7487599710564+0\right.\right.$. $^{\prime}$

i் $)+\left(\left(157.63997123695722{ }_{-}+0\right.\right.$. $\left.\left.\left.\left.\left.\left.\dot{\mathbb{1}}\right)-\left(31.01317766069574{ }_{-}+0{ }^{`} \dot{\mathbb{1}}\right) \alpha \mathrm{d} 0\right) \alpha \mathrm{d} 0\right)\right)\right)\right)+\mathrm{k} 1^{5} \alpha \mathrm{d} 0^{2}$ $\left(\left(0 .^{\prime}+1098.0025115996561^{`} \dot{\mathbb{1}}\right)+\mathrm{k} 2^{2}\left(\left(0{ }^{\prime}{ }_{-}+2.9621333664 \dot{\mathrm{i}}\right)+\alpha \mathrm{d} 0\right.\right.$

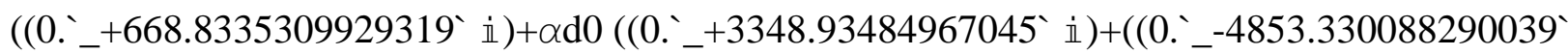

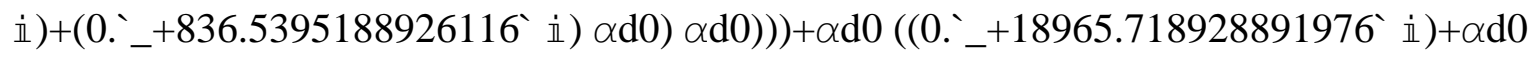
$\left(\left(0 .{ }_{-}-32458.549517936146\right.\right.$ i் $)+\alpha \mathrm{d} 0\left(\left(0{ }^{\prime} \_+11898.828269937325\right.\right.$ i $)+\alpha \mathrm{d} 0$ $\left(\left(0 ._{-}+682.213850269705\right.\right.$ i $)-\left(0 .{ }_{-}+166.25339616741059 ` \dot{1}\right)$

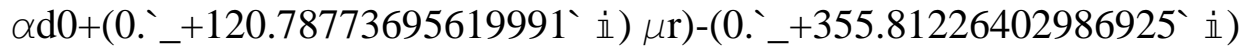

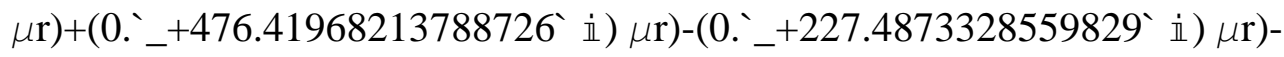
$\left.\left(0 .{ }_{-}+14.249016357409598^{`} \dot{i}\right) \mu \mathrm{r}\right)+\mathrm{k} 1 \mathrm{k} 2^{4} \alpha \mathrm{d} 0^{2}\left(\left(7.958078640513122^{` *}{ }^{\prime}{ }_{-}\right.\right.$

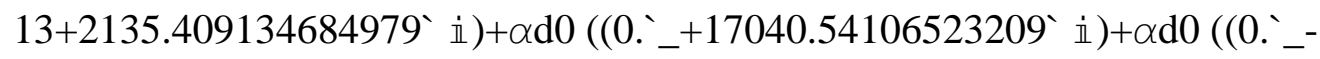

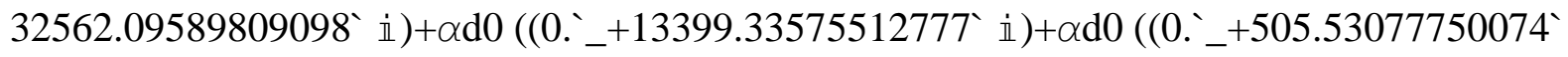

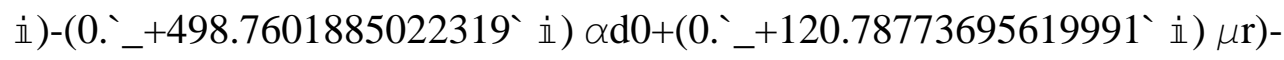
$\left(0 .{ }_{-}+355.8122640298693\right.$ ii $\left.) \mu \mathrm{r}\right)+\left(0 .{ }_{-}+476.4196821378873\right.$ i $\left.) \mu \mathrm{r}\right)-$ $\left(0 ._{-}+227.4873328559829\right.$ i 1$\left.\left.) \mu \mathrm{r}\right)-\left(0 .^{\prime}+14.249016357409598^{`} \dot{\mathrm{i}}\right) \mu \mathrm{r}\right)+\mathrm{k} 1^{3} \mathrm{k} 2^{2} \alpha \mathrm{d} 0^{2}$

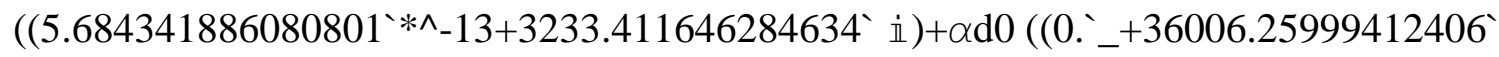

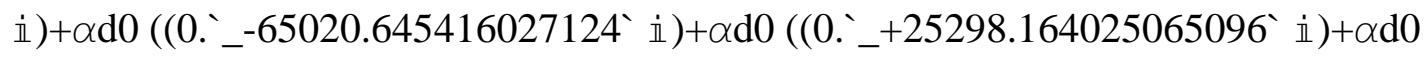

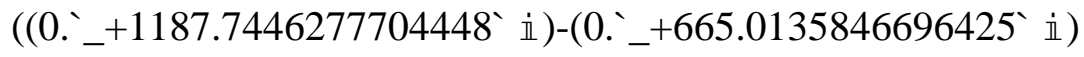

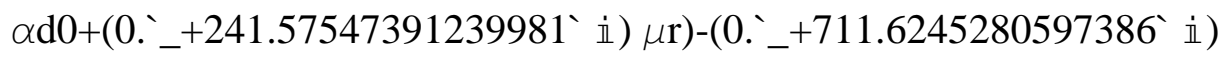

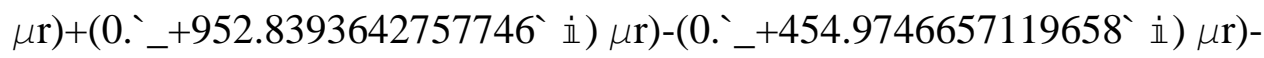

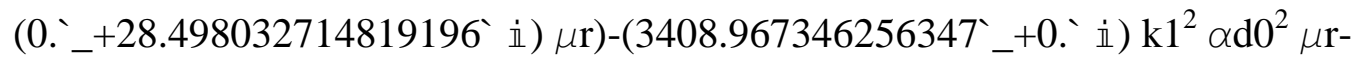
(3408.967346256347_+0. $\dot{\mathbb{1}}) \mathrm{k}^{2} \alpha \mathrm{d} 0^{2} \mu \mathrm{r}+\left(3404.7667553568326{ }_{-}+0{ }^{`} \dot{\mathbb{1}}\right) \mathrm{k} 1^{2} \alpha \mathrm{d} 0^{3}$

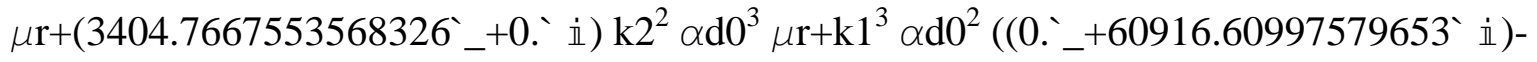
$\left(0 .{ }_{-}+2069.57558698786\right.$ i $) \mu \mathrm{r}+\alpha \mathrm{d} 0\left(\left(0\right.\right.$. $_{-}-121683.89096164228{ }^{\prime}$ 


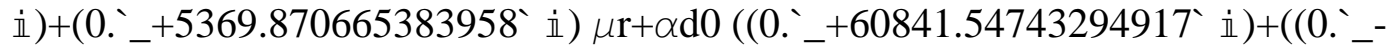
4950.950280959649 立 $)+\left(0 ._{-}+1648.1165395161072\right.$ i $\left.\left.\left.\left.) \alpha \mathrm{d} 0\right) \mu \mathrm{r}\right)\right)\right)+\mathrm{k} 1 \mathrm{k} 2^{2} \alpha \mathrm{d} 0^{2}$

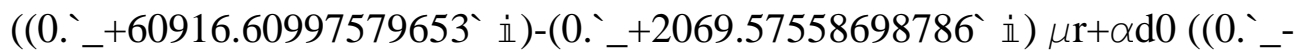

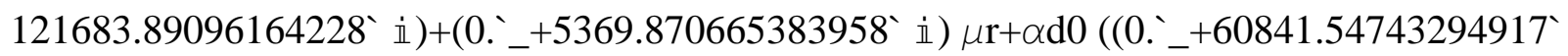

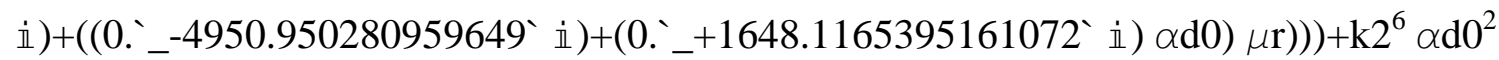
((3.506338886762477_+0`立)-(0.030818359999999996`_+0`立) $\mu \mathrm{r}+\alpha \mathrm{d} 0$ ((934.6035434939477_+0.’ $\dot{\mathbb{1}})-\left(7.8131641761873905_{-}+0\right.$.`立 $) \mu \mathrm{r}+\alpha \mathrm{d} 0$ ((3539.5018891629447_+0`立)-(66.97035006804725_+0.`立) $\mu \mathrm{r}+\alpha \mathrm{d} 0((-$

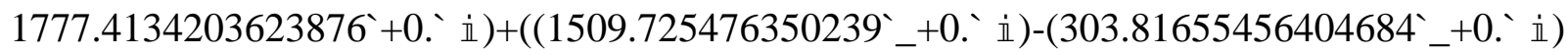

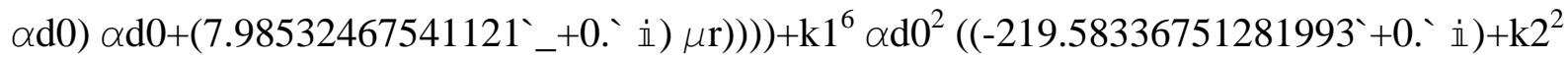
$\left(\left(0.0031999999999999997 \_+0{ }^{`} \dot{1}\right)+\alpha \mathrm{d} 0\left(\left(1.52573371596800022_{-}+0{ }^{`} \dot{1}\right)+\alpha \mathrm{d} 0\right.\right.$ $\left.\left.\left(\left(183.131640028595 \_+0 ` \dot{1}\right)+\left(748.5369706660108^{\prime}+0{ }^{`} \dot{1}\right) \alpha \mathrm{d} 0\right)\right)\right)-$

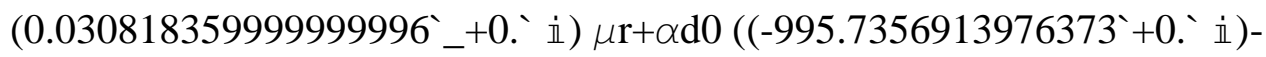

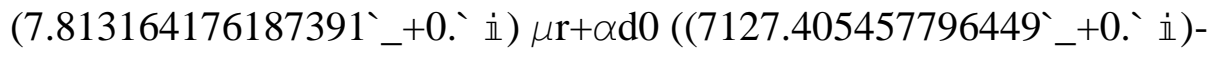
(66.97035006804724__+0`立) $\mu \mathrm{r}+\alpha \mathrm{d} 0((-1495.0350159950144 `+0$ i $)+\alpha \mathrm{d} 0((-$ $989.145196755006+0$.

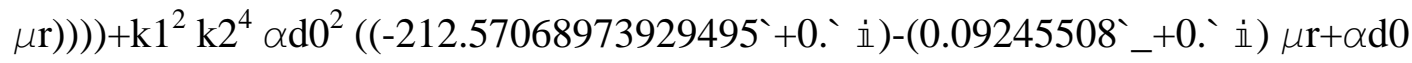

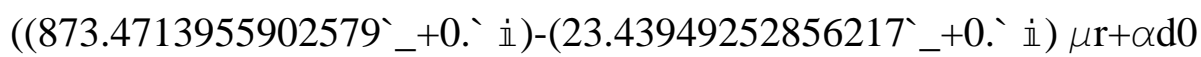
((14206.40923612234__+0`立)-(200.91105020414176__+0`立) $\mu \mathrm{r}+\alpha \mathrm{d} 0((-$ $5049.86185671979+0 . ` \dot{1})+\left(\left(2030.3057559454724{ }_{-}+0\right.\right.$.

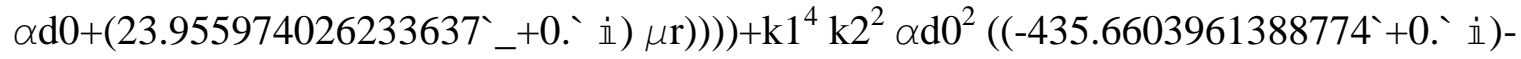

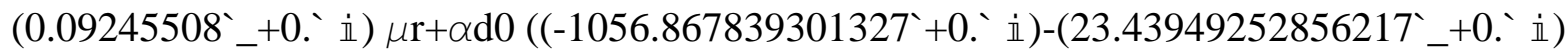
$\mu \mathrm{r}+\alpha \mathrm{d} 0\left(\left(17794.3128047558422_{-}+0{ }^{`} \dot{\mathbb{1}}\right)-\left(200.91105020414176{ }_{-}+0\right.\right.$ 。' $\left.\dot{\mathbb{1}}\right) \mu \mathrm{r}+\alpha \mathrm{d} 0((-$ $4767.483452352417+0$ ＇立 $)+\alpha \mathrm{d} 0((-468.56491715977256$ +0`立 $)+(652.5846326948243$ _ +0 . i) $\alpha \mathrm{d} 0)+\left(23.955974026233637 \_+0\right.$.'这 $\left.\left.\left.\left.\mu \mathrm{r}\right)\right)\right)\right)+\mathrm{k} 1^{4} \alpha \mathrm{d} 0^{2}((-14880.053844279264+0$.'

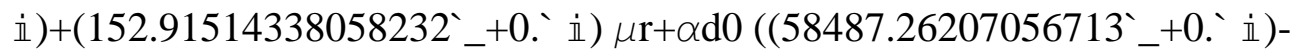

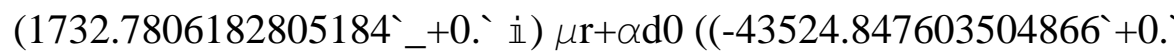
$\dot{\mathbb{1}})+\left(1437.066396887926 \_+0\right.$.

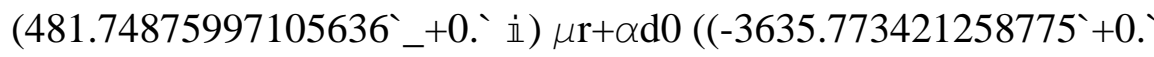

í $)+\left(\left(157.63997123695725{ }_{-}+0\right.\right.$. $\left(\left(-11367.385321602256+00^{`} \dot{i}\right)+\left(90.93756473002034 \_+0\right.\right.$ ` $\left.^{2}\right) \mu \mathrm{r}+\alpha \mathrm{d} 0$ $\left(\left(93833.18528097369{ }^{`}+0{ }^{`} \dot{1}\right)+c r 0\left(\left(-17261.21869527362 `+0{ }^{`}\right.\right.\right.$

$\dot{\mathbb{1}})+\left((25854.616092713557\right.$ _+0.`立 $)-\left(8604.051834033518^{\prime}+0\right.$.’ $\left.\left.\left.\dot{\mathbb{1}}\right) \alpha \mathrm{d} 0\right) \alpha \mathrm{d} 0\right)-$ $(2838.7940692466555$ _ +0 .' $\dot{\mathbb{1}}) \mu \mathrm{r}+\alpha \mathrm{d} 0\left(\left(-111767.73443363383{ }^{`}+0\right.\right.$. $^{\prime}$

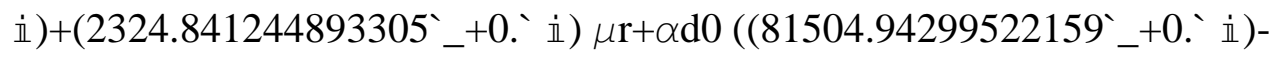
(947.788616872645_+0.' $\dot{\mathbb{1}}) \mu \mathrm{r}+\alpha \mathrm{d} 0\left(\left(-23136.5434515913{ }^{`}+0{ }^{`}\right.\right.$

$\dot{\mathbb{1}})+\left(499.01449836150266\right.$ _+0.'立 $\left.\left.\left.\left.\left.\mu \mathrm{r}-\left(124.05271064278297{ }_{-}+0{ }^{`} \dot{\mathbb{1}}\right) \alpha \mathrm{d} 0 \mu \mathrm{r}\right)\right)\right)\right)\right)+\mathrm{k} 2^{4} \alpha \mathrm{d} 0^{2}$ ((3512.6685226770073`_+0`立)-(61.97757865056198`_+0`立) $\mu \mathrm{r}+\alpha \mathrm{d} 0$ $\left(\left(35345.92321040656 \_+0{ }^{`} \dot{1}\right)+c r 0\left(\left(-17261.21869527362 `+0{ }^{`}\right.\right.\right.$

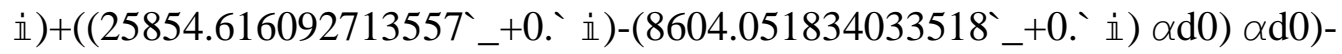


$\left(1106.0134509661368{ }^{\prime}+0 . ` \dot{1}\right) \mu \mathrm{r}+\alpha \mathrm{d} 0\left(\left(-68242.88683012896+00^{`}\right.\right.$

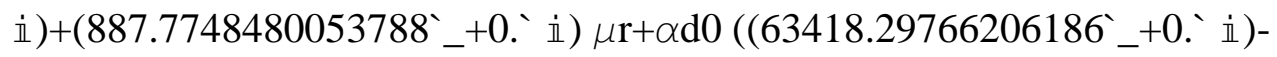
(466.0398569015887_+0``i $) \mu \mathrm{r}+\alpha \mathrm{d} 0\left(\left(-19500.770030332525{ }^{\circ}+0\right.\right.$.

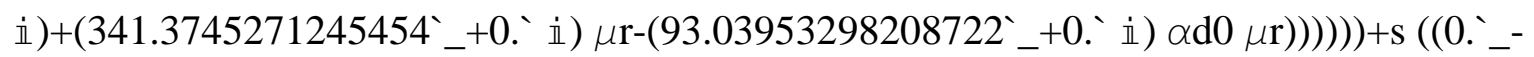

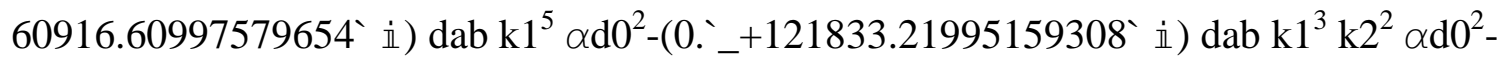

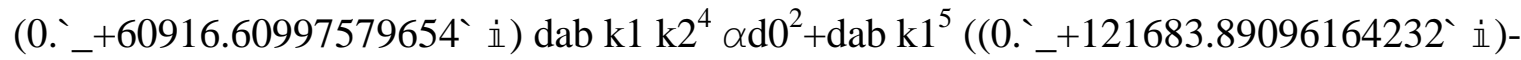

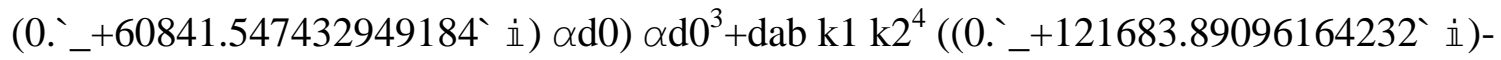

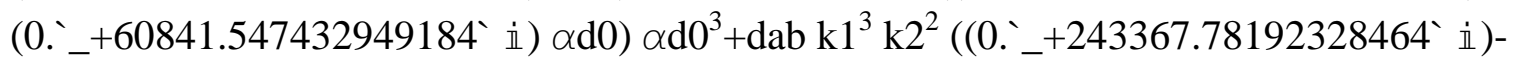
$\left(0 .{ }_{-}+121683.09486589837\right.$ i $\left.) \alpha \mathrm{d} 0\right) \alpha \mathrm{d} 0^{3}+\mathrm{dab} \mathrm{k} 1^{6} \mathrm{k}^{4} \alpha \mathrm{d} 0^{2}\left(\left(-0.008^{\circ}+0 . ` \dot{1}\right)+\alpha \mathrm{d} 0((-\right.$

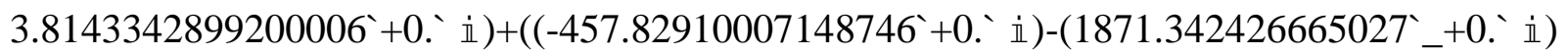

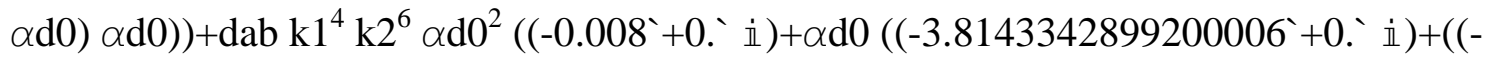

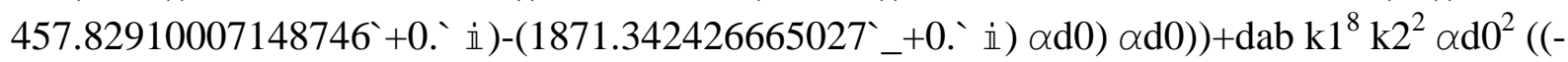

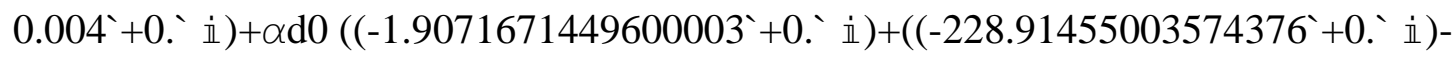
(935.6712133325135_+0.`i $) \alpha \mathrm{d} 0) \alpha \mathrm{d} 0))+\mathrm{dab} \mathrm{k}^{2} \mathrm{k}^{8} \alpha \mathrm{d} 0^{2}((-0.004+0 . ` \dot{1})+\alpha \mathrm{d} 0((-$

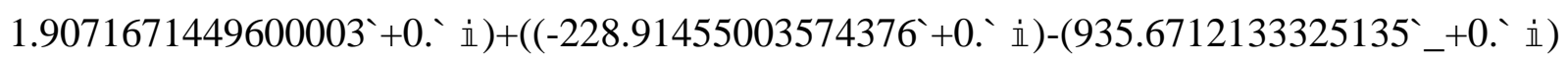

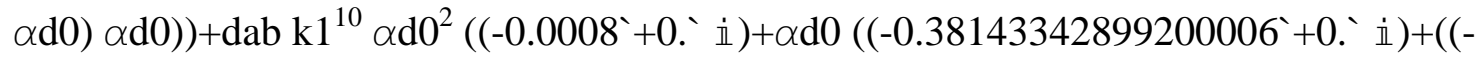

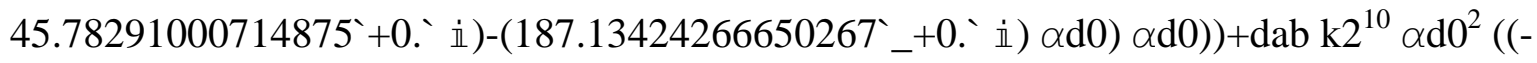

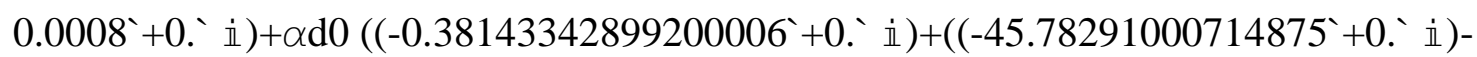

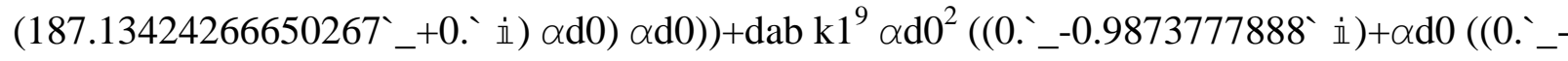

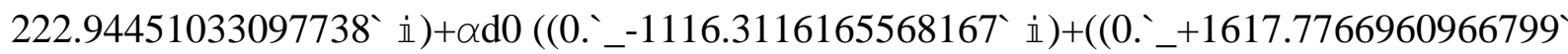

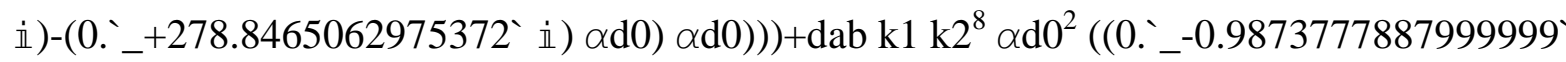
ii $)+\alpha \mathrm{d} 0\left(\left(0 ._{-}{ }_{-}-222.9445103309773 ` \dot{\mathrm{i}}\right)+\alpha \mathrm{d} 0\left(\left(0 ._{-}-1116.3116165568167^{\circ}\right.\right.\right.$

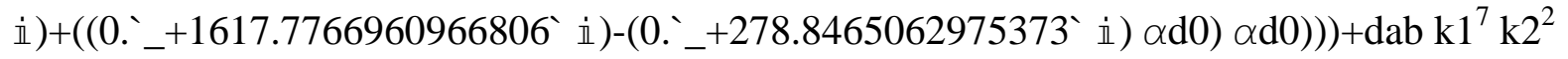

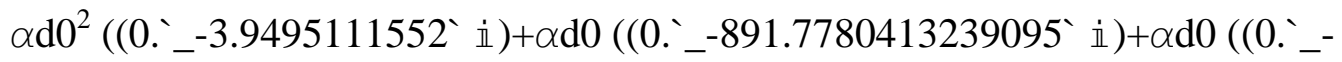
4465.246466227267 ì $)+\left(\left(0 ._{-}+6471.10678438672 ` \dot{i}\right)-\left(0 ._{-}+1115.3860251901485\right.\right.$ i $\left.) \alpha \mathrm{d} 0\right)$

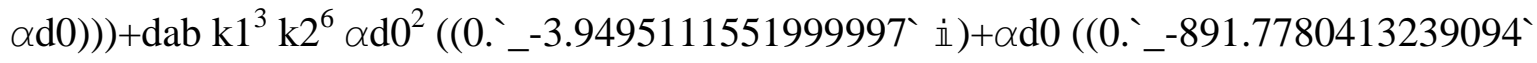

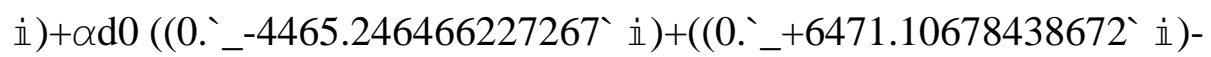

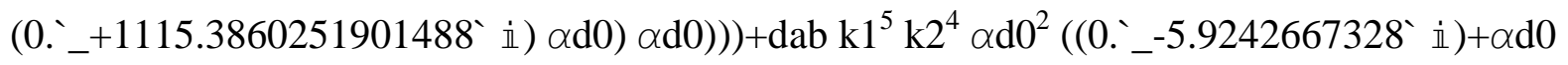
$\left(\left(0 .{ }^{\prime}-1337.6670619858642 ` i \mathrm{i}\right)+\alpha \mathrm{d} 0\left(\left(0 .^{\prime}-6697.8696993408999^{\circ}\right.\right.\right.$

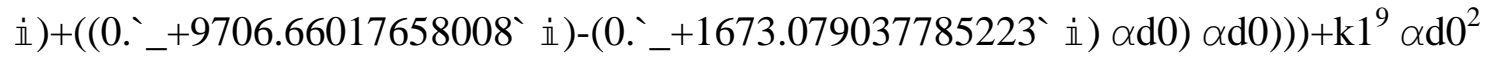
$\left(\left(0 .{ }_{-}+0.014144\right.\right.$ i) $)+\alpha \mathrm{d} 0\left(\left(0 .{ }_{-}+6.0365430245785605\right.\right.$ i $)+\alpha \mathrm{d} 0\left(\left(0 .{ }_{-}+653.6815888877337\right.\right.$

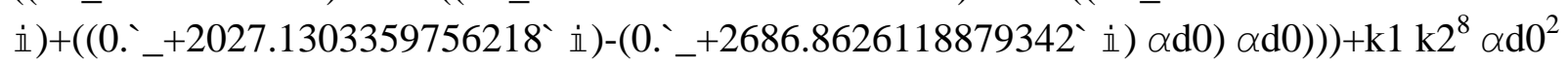

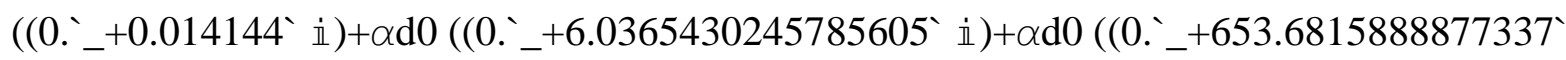

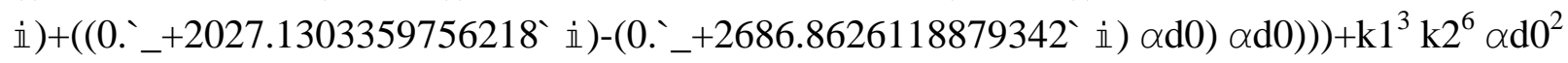

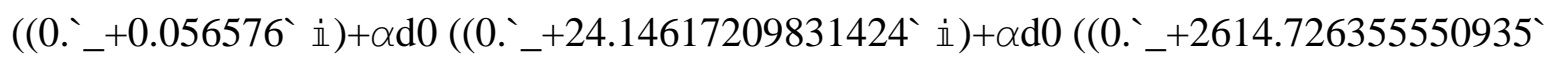

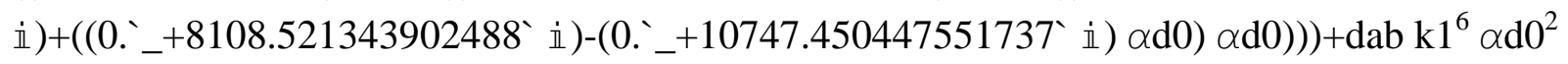

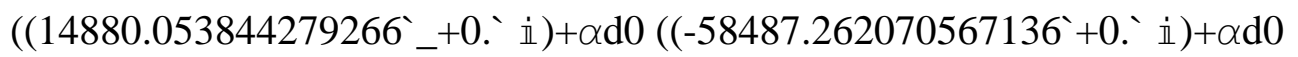
((43524.84760350488_+0.`i $)+\alpha \mathrm{d} 0\left(\left(-18086.64533315973{ }^{`}+0\right.\right.$.

i $\left.\left.\left.\left.)+\left(3635.773421258775 \_+0 . ` \dot{1}\right) \alpha \mathrm{d} 0\right)\right)\right)\right)+\mathrm{dab} \mathrm{k} 2^{6} \alpha \mathrm{d} 0^{2}((-3512.6685226770073 `+0 . \grave{1})+\alpha \mathrm{d} 0$

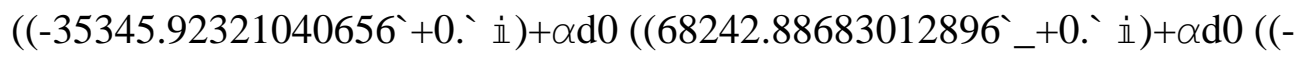




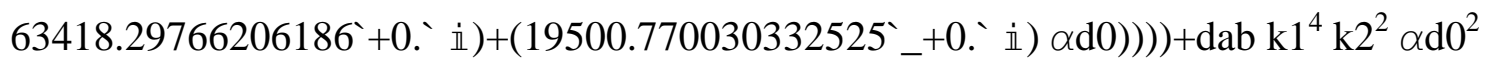
$\left(\left(26247.439165881522 `+0 . ` i \frac{1}{)}+\alpha \mathrm{d} 0((-152320.44735154085+0 . ` \dot{\mathrm{i}})+\alpha \mathrm{d} 0\right.\right.$

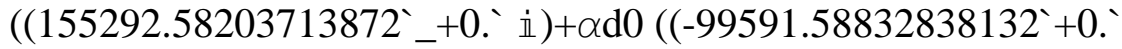

i் $\left.\left.\left.\left.)+\left(26772.316872850075 \_+0 . ` \dot{\mathrm{i}}\right) \alpha \mathrm{d} 0\right)\right)\right)\right)+\mathrm{dab} \mathrm{k}^{2} \mathrm{k}^{4} \alpha \mathrm{d} 0^{2}\left(\left(7854.71679892525 \_+0\right.\right.$.

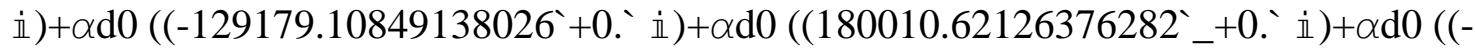

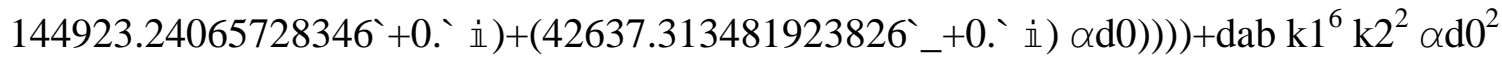

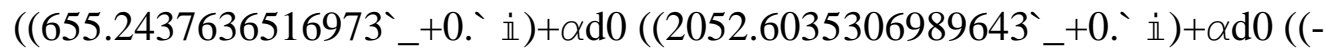

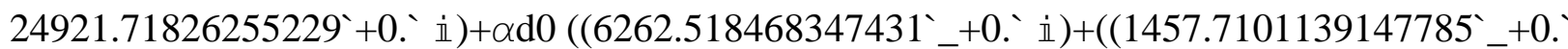

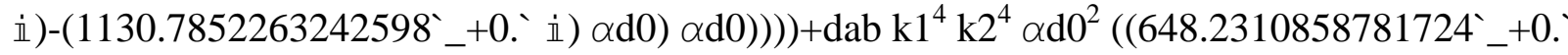

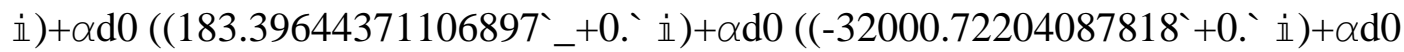

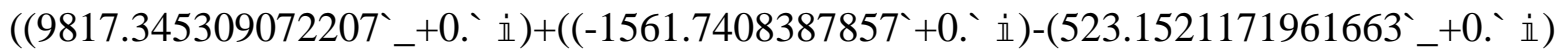
$\alpha \mathrm{d} 0) \alpha \mathrm{d} 0))))+\mathrm{dab} \mathrm{k1}{ }^{8} \alpha \mathrm{d} 0^{2}\left(\left(219.58336751281993 \_+0 . ` \dot{i}\right)+\alpha \mathrm{d} 0\left(\left(995.7356913976374 \_+0 .\right.\right.\right.$ $\dot{1})+\alpha \mathrm{d} 0\left(\left(-7127.4054577964477^{\circ}+0{ }^{`} \dot{1}\right)+\alpha \mathrm{d} 0((1495.0350159950149 `+0\right.$.

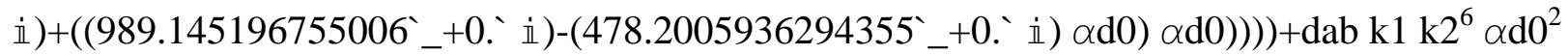
$\left(\left(0 .{ }_{-}-2135.409134684979\right.\right.$ i $)+\alpha \mathrm{d} 0\left(\left(0 ._{-}-17040.54106523209\right.\right.$ i $)+\alpha \mathrm{d} 0$

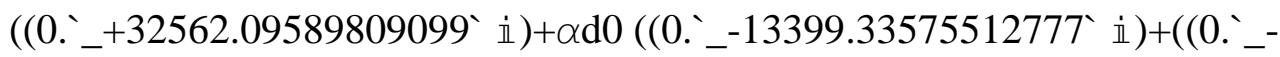
505.53077750074044 i $\left.\left.\left.\left.\left.)+\left(0 .{ }_{-}+498.7601885022318 ` \dot{i}\right) \alpha \mathrm{d} 0\right) \alpha \mathrm{d} 0\right)\right)\right)\right)+\mathrm{dab} k 2^{8} \alpha \mathrm{d} 0^{2}((-$ $3.506338886762477+0 . ` \dot{1})+\alpha \mathrm{d} 0\left(\left(-934.6035434939477{ }^{\circ}+0 ` \dot{1}\right)+\alpha \mathrm{d} 0((-\right.$ $3539.501889162945+0$.`立 $)+\alpha \mathrm{d} 0\left(\left(1777.4134203623873{ }_{-}+0 . ` \dot{1}\right)+\alpha \mathrm{d} 0((-\right.$ $1509.725476350239 `+0$ ‘

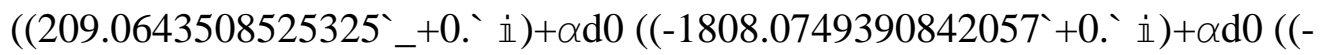
$17745.91112528528+0$.`立 $)+\alpha \mathrm{d} 0\left(\left(6827.275277082176 \_+0\right.\right.$.`立 $)+\alpha \mathrm{d} 0((-$

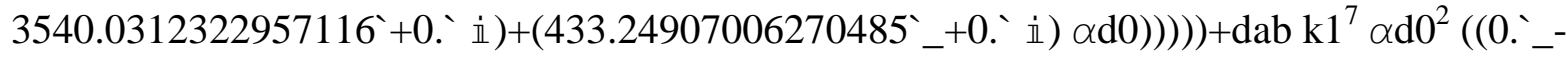
1098.0025115996561 i $)+\alpha \mathrm{d} 0\left(\left(0{ }^{\prime}-18965.718928891976\right.\right.$ i $)+\alpha \mathrm{d} 0$

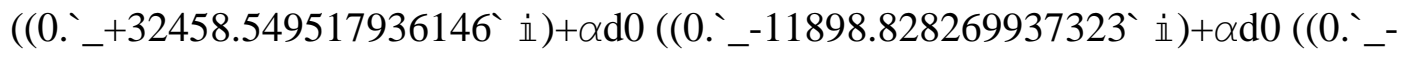
682.2138502697048 i $)+\left(\left(0 .{ }_{-}+166.25339616741036 ` \dot{i}\right)+\left(0 .{ }_{-}+3.05299906844081^{*} * \Lambda_{-} 14\right.\right.$ i $)$

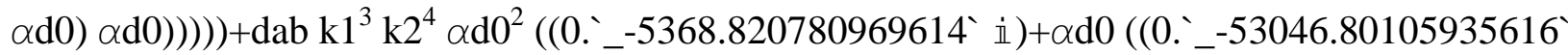
i $)+\alpha \mathrm{d} 0\left(\left(0 .{ }_{-}+97582.74131411812\right.\right.$ i $)+\alpha \mathrm{d} 0\left(\left(0 .{ }_{-}-38697.49978019286\right.\right.$ i $)+\alpha \mathrm{d} 0\left(\left(0 .{ }_{-}-\right.\right.$ 1693.2754052711857 i $)+\left(\left(0 .{ }_{2}+1163.773773171874\right.\right.$ i $)+\left(0 .{ }_{-}+3.05299906844081^{*} * \Lambda_{-} 14\right.$ i $)$ $\alpha \mathrm{d} 0) \alpha \mathrm{d} 0)))))+\mathrm{dab} \mathrm{k1}{ }^{5} \mathrm{k} 2^{2} \alpha \mathrm{d} 0^{2}\left(\left(0 ._{-}^{\prime}-4331.414157884291^{`} \dot{i}\right)+\alpha \mathrm{d} 0\left(\left(0 ._{-}^{\prime}-\right.\right.\right.$

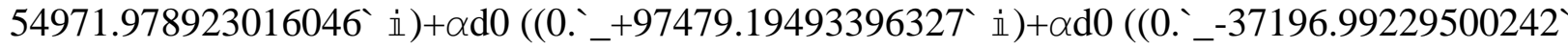
i $)+\alpha \mathrm{d} 0\left(\left(0 .{ }_{-}-1869.95847804015 ` \dot{\mathrm{i}}\right)+\left(\left(0 .{ }_{-}+831.2669808370524\right.\right.\right.$ i $)+\left(0 .{ }^{\prime}+6.10599813688162^{*} *{ }^{-1}-14\right.$ i $\left.\left.\left.\left.\left.\left.) \alpha \mathrm{d} 0\right) \alpha \mathrm{d} 0\right)\right)\right)\right)\right)+\mathrm{k} 1^{4} \mathrm{k} 2^{4} \alpha \mathrm{d} 0^{2}((-23.47068711936+0$.

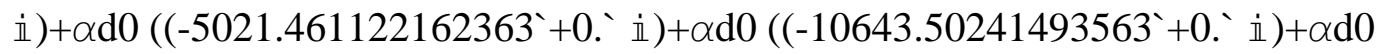
((27996.198832227536_+0.`i $)-(12307.764608010182 `+0 . ` i) ~ \alpha \mathrm{d} 0-$ (1122.8054559990162`_+0.'i $) \mu \mathbf{r})-\left(274.6974600428925 \_+0 `\right.$ i $\left.) \mu \mathbf{r}\right)-$

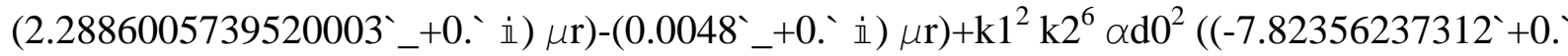

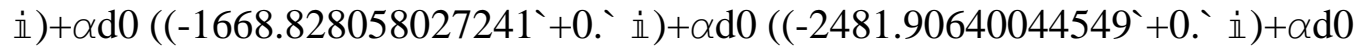

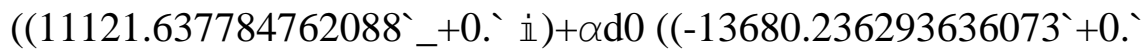
ii $)+(6717.156529719836$ _+0.`i $) \alpha \mathrm{d} 0)-(748.5369706660108$ _+0.`i $) \mu \mathrm{r})-$ 
(183.131640028595_+0.`立) $\mu \mathrm{r})-\left(1.5257337159680002 \_+0\right.$ ‘这 $\left.\mu \mathrm{r}\right)-$ $\left.\left(0.0031999999999999997 \_+0 . ` \dot{i}\right) \mu \mathrm{r}\right)+\mathrm{k} 1^{8} \alpha \mathrm{d} 0^{2}((-7.82356237312 `+0 ` \dot{i})+\alpha \mathrm{d} 0((-$

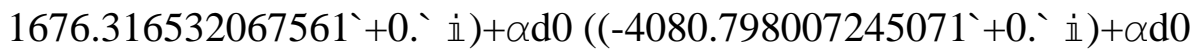

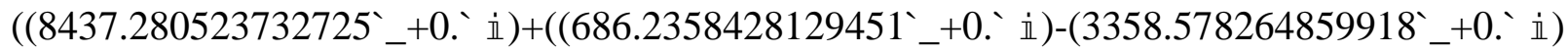
$\alpha \mathrm{d} 0) \alpha \mathrm{d} 0-\left(187.13424266650267 \_+0 . ` i\right.$ i) $\left.\left.\mu \mathrm{r}\right)-\left(45.78291000714875 \_+0 . ` i \mathbf{i}\right) \mu \mathrm{r}\right)-$

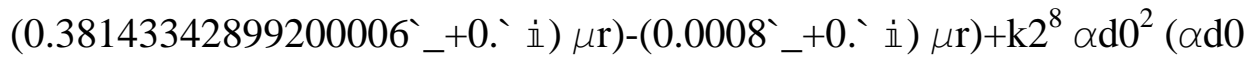
$\left(\left(2.49615801344 \_+0\right.\right.$.` $\left.\dot{i}\right)+\alpha \mathrm{d} 0\left(\left(532.9638689331938 \_+0 . ` \dot{1}\right)+\alpha \mathrm{d} 0\right.$ $\left(\left(894.7857536764543{ }^{\prime}+0 . ` i \mathrm{i}\right)+\alpha \mathrm{d} 0((-4788.824045483007+0\right.$ ○

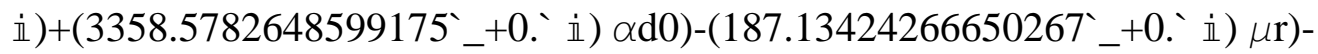

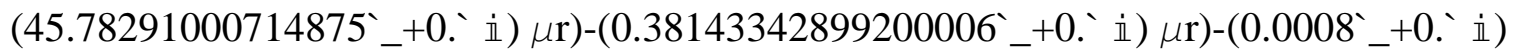

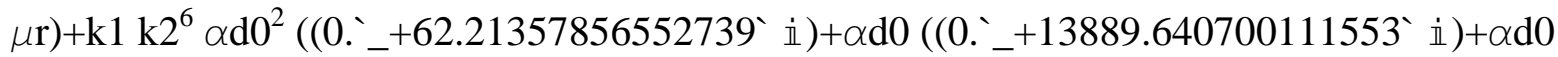

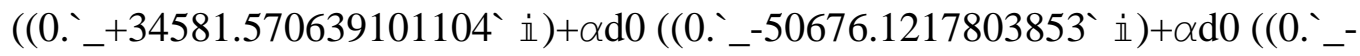

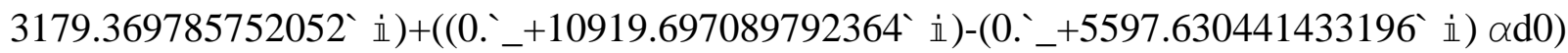
$\alpha \mathrm{d} 0-\left(0 .^{\prime}+278.8465062975373\right.$ i $\left.) \mu \mathrm{r}\right)+\left(0 .^{\prime}+1617.7766960966806\right.$ ii $\left.) \mu \mathrm{r}\right)-$ $\left(0 . \_+1116.3116165568167\right.$ i $\left.) \mu \mathrm{r}\right)-\left(0 .{ }_{-}+222.9445103309773\right.$ i $\left.) \mu \mathrm{r}\right)-$

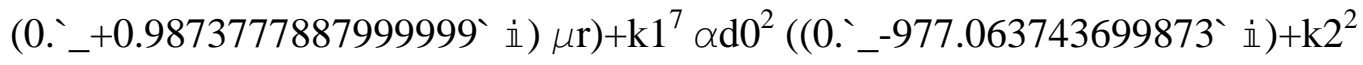

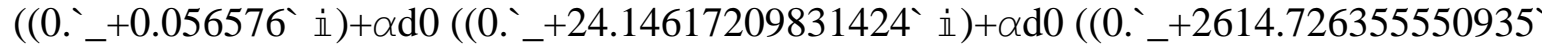

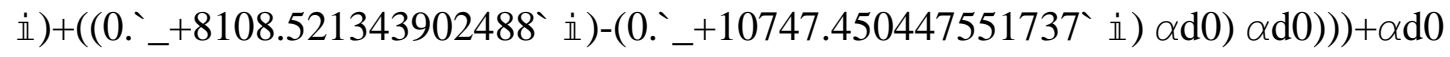
$\left(\left(0 .^{\prime}+11401.562491238034\right.\right.$ i $)+\alpha \mathrm{d} 0\left(\left(0 .^{\prime}+38520.26714096152 ` i \mathrm{i}\right)+\alpha \mathrm{d} 0\left(\left(0 ._{-}-\right.\right.\right.$

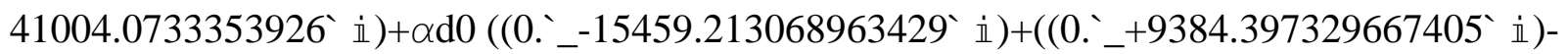
$\left(0 .{ }_{-}+1865.8768138110654\right.$ ii) $\left.\alpha \mathrm{d} 0\right) \alpha \mathrm{d} 0-\left(0 ._{-}+278.8465062975372 ` i\right.$ i)

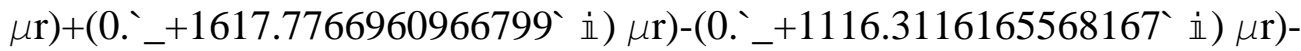

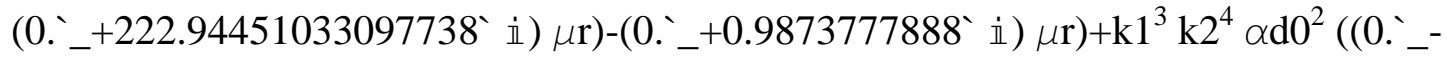
852.6365865688183 i $)+\alpha \mathrm{d} 0\left(\left(0 `_{-}+39180.84389146114 i \dot{i}\right)+\alpha \mathrm{d} 0\right.$

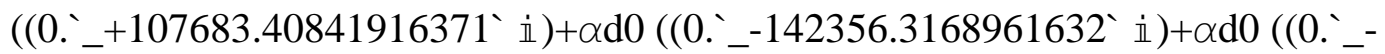
21817.952640467534 i $)+\left(\left(0 .{ }_{-}+31223.791509252136\right.\right.$ i $)-\left(0 .{ }_{-}+13061.137696677459\right.$ i $)$

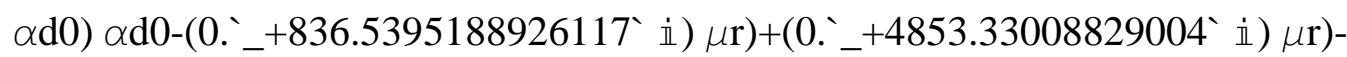
$\left(0 ._{-}+3348.93484967045\right.$ i) $\left.\mu \mathrm{r}\right)-\left(0 .{ }^{\prime}+668.8335309929321\right.$ i $\left.) \mu \mathrm{r}\right)-\left(0 .{ }_{-}+2.9621333664\right.$ i $)$ $\mu \mathrm{r})+\mathrm{k} 1^{3} \alpha \mathrm{d} 0^{2}\left(\left(0 ._{-}^{\prime}-60916.60997579654\right.\right.$ i $)+\left(\left(0 .^{\prime}+121683.89096164232 ` \dot{1}\right)-\right.$ $\left(0 .{ }_{-}+60841.547432949184\right.$ i $\left.\left.\left.\dot{1}\right) \alpha \mathrm{d} 0\right) \alpha \mathrm{d} 0\right) \mu \mathrm{r}+\mathrm{k} 1 \mathrm{k} 2^{2} \alpha \mathrm{d} 0^{2}\left(\left(0 .{ }_{-}-60916.60997579654\right.\right.$

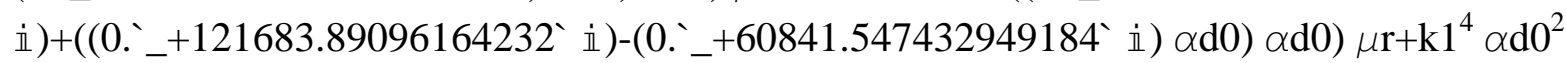

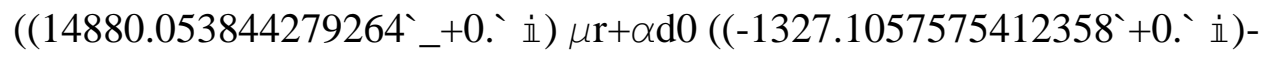
(58487.26207056713__+0`i) $\mu \mathrm{r}+\alpha \mathrm{d} 0\left(\left(1327.1057575412358 \_+0 .\right.\right.$ i) $+\left(\left(43524.847603504866 \_+0\right.\right.$. $\left.\dot{1}\right)+\alpha \mathrm{d} 0\left(\left(-18086.645333159722{ }^{\circ}+0\right.\right.$.

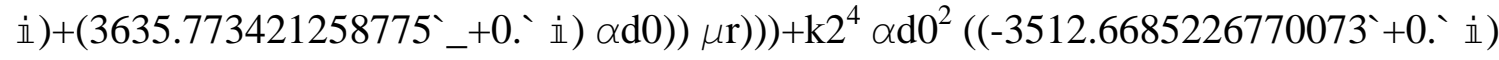

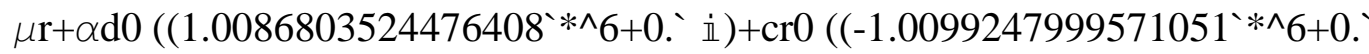
i் $)+\left(1.0086803524476407^{* \wedge} 6+0\right.$.`这 $\left.) \alpha \mathrm{d} 0\right)-(35345.92321040656$ _ $+0 . ` \dot{1}) \mu \mathrm{r}+\alpha \mathrm{d} 0((-$ $2.016117790816429 * \wedge 6+0$.'i $)+(68242.88683012896$ _ +0 .`i $) \mu \mathbf{r}+\alpha \mathrm{d} 0$

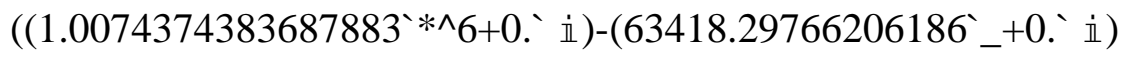
$\left.\left.\left.\left.\mu \mathrm{r}+\left(19500.770030332525 \_+0 .^{`} \dot{\mathrm{i}}\right) \alpha \mathrm{d} 0 \mu \mathrm{r}\right)\right)\right)\right)+\mathrm{k} 1^{2} \mathrm{k} 2^{2} \alpha \mathrm{d} 0^{2}\left(\left(11367.385321602256 \_+0 . ` \dot{\mathrm{i}}\right)\right.$ 
$\mu \mathrm{r}+\alpha \mathrm{d} 0\left(\left(1.00735324669009966^{* \wedge} 6+0{ }^{`} \dot{1}\right)+\operatorname{cr} 0\left(\left(-1.0099247999571051^{` * \wedge} 6+0\right.\right.\right.$.

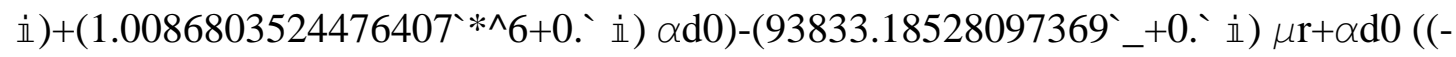
$2.0147906850588878^{` * \wedge} 6+0$. $\left.\dot{\mathbb{1}}\right)+\left(111767.73443363383^{`}+0 . ` \dot{\mathbb{1}}\right) \mu \mathrm{r}+\alpha \mathrm{d} 0$

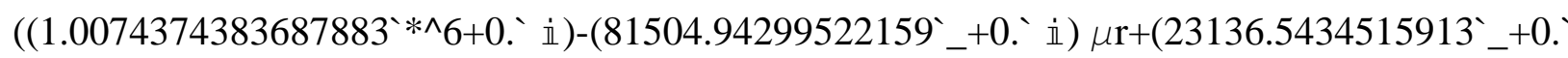

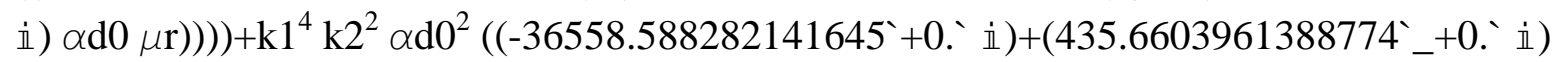
$\mu \mathrm{r}+\alpha \mathrm{d} 0\left(\left(-44711.81128337758 `+0\right.\right.$ ` $\left.^{\mathrm{i}}\right)+\mathrm{cr} 0\left(\left(-8813.665728477792 `+0\right.\right.$ ` $\left.^{\mathrm{i}}\right)+\alpha \mathrm{d} 0((-$ $64394.90003199727+0$. $\dot{\mathbb{1}})+(73107.5098733866$ _+0.'立 $\alpha \mathrm{d} 0))+(1056.8678393013267$ _ +0 .

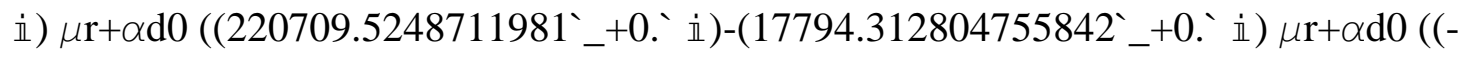

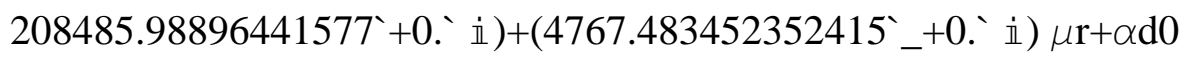

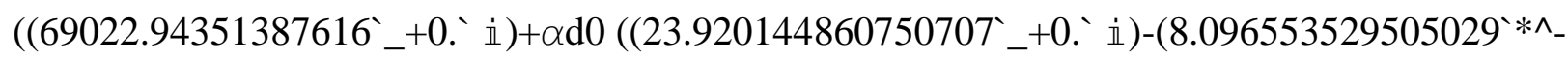

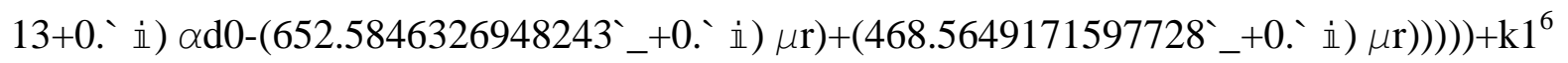

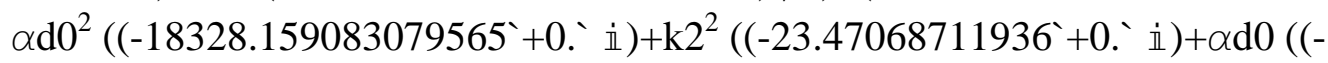
$5026.453438189243 `+0 ` \dot{1})+\alpha \mathrm{d} 0((-11709.430152802019 `+0 ` \dot{1})+\alpha \mathrm{d} 0$

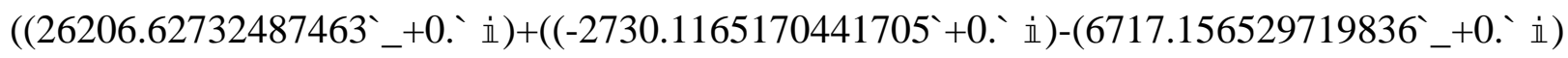

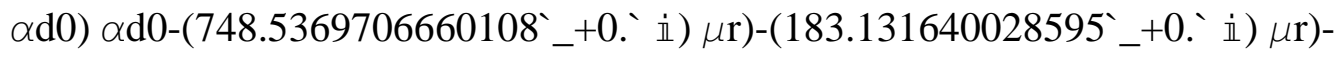

$\left.\left(1.5257337159680002 \_+0 . \grave{1}\right) \mu \mathrm{r}\right)-\left(0.0031999999999999997 \_+0 . \dot{1}\right)$

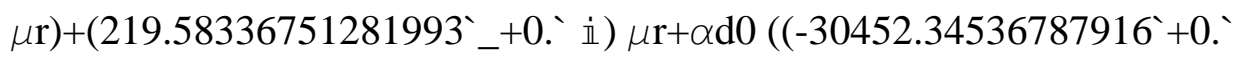

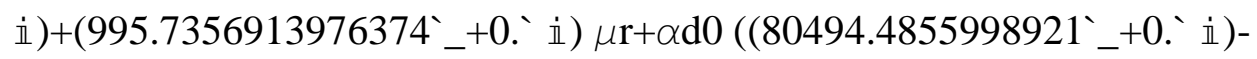
(7127.405457796447_+0`立) $\mu \mathrm{r}+\alpha \mathrm{d} 0\left(\left(-12334.20954883405{ }^{`}+0\right.\right.$.

$\dot{\mathbb{1}})+(1495.0350159950149$ _+0`立 $) \mu \mathrm{r}+\alpha \mathrm{d} 0((-19385.751636314508 `+0$ ‘ $\dot{\mathbb{1}})+\alpha \mathrm{d} 0$ ((5.980036215187351__+0`立)-(8.096553529505029`*^-13+0.`立) $\alpha \mathrm{d} 0-$

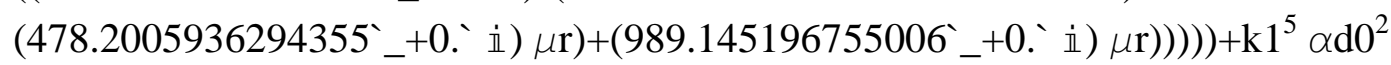

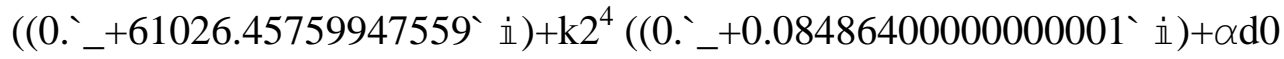
$\left(\left(0 ._{-}+36.21925814747136\right.\right.$ i $)+\alpha \mathrm{d} 0\left(\left(0{ }^{\prime}{ }_{-}+3922.0895333264025\right.\right.$

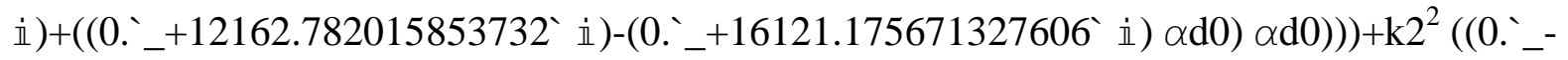
$\left.1891.9139088342188^{`} \dot{\mathbb{i}}\right)+\alpha \mathrm{d} 0\left(\left(0\right.\right.$.`_$\left._{-}+36692.76568258762 ` \dot{1}\right)+\alpha \mathrm{d} 0$

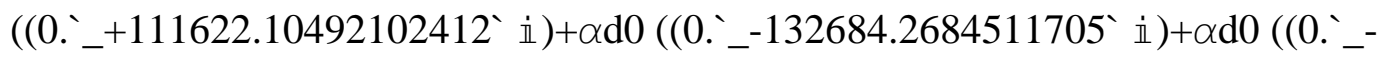

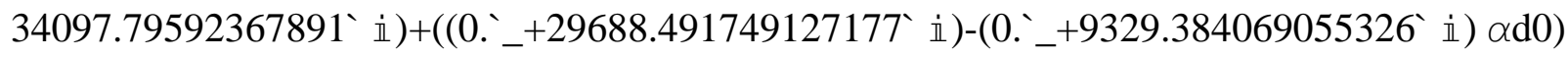

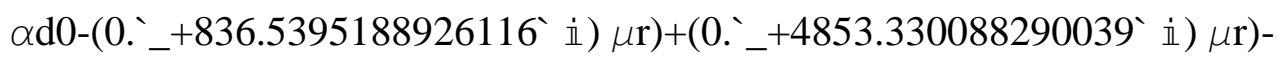

$\left(0 .{ }^{\prime}+3348.93484967045\right.$ in $\left.\left.) \mu \mathrm{r}\right)-\left(0 ._{-}+668.8335309929321^{`} \dot{\mathbb{1}}\right) \mu \mathrm{r}\right)-$

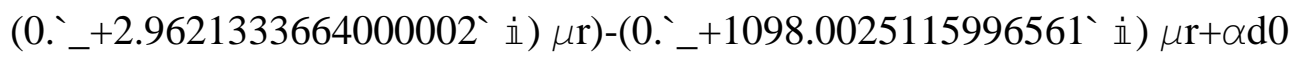

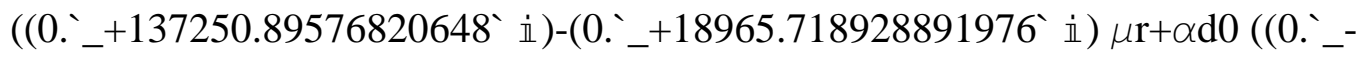

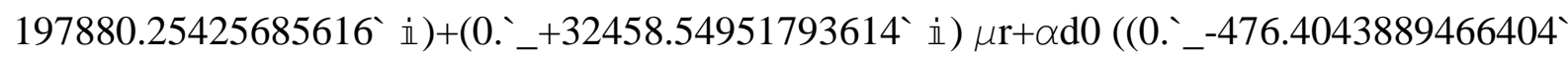

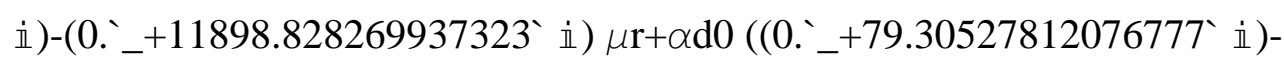

$\left(0 ._{-}+682.213850269705\right.$ 立 $\left.\left.\left.\left.\left.) \mu \mathrm{r}+\left(0 ._{-}+166.25339616741059 ` \dot{1}\right) \alpha \mathrm{d} 0 \mu \mathrm{r}\right)\right)\right)\right)\right)+\mathrm{k} 1 \mathrm{k} 2^{4} \alpha \mathrm{d} 0^{2}$ $\left(\left(1.8189894035458565^{` * \wedge}-11+61026.45759947557\right.\right.$ i $)-\left(0 `^{\prime}+2135.409134684979 ` \dot{1}\right) \mu \mathrm{r}+\alpha \mathrm{d} 0$

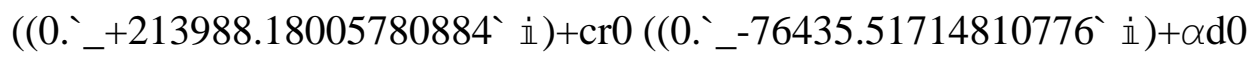

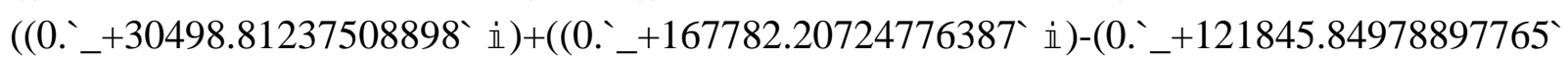

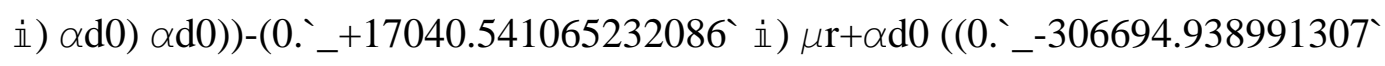

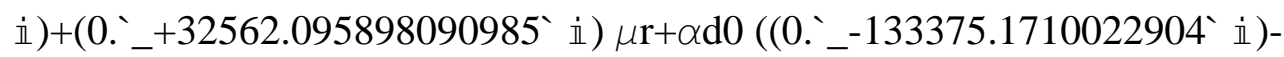


$\left(0 . \_+13399.33575512777\right.$ i $) \mu \mathrm{r}+\alpha \mathrm{d} 0\left(\left(00_{-}+285373.07834052277\right.\right.$ i $)-$

$\left(0 . \_+120317.60600420985\right.$ ii $) \alpha \mathrm{d} 0-\left(0 .{ }_{-}+505.53077750074044\right.$ i $)$ $\mu \mathrm{r}+\left(0 ._{-}+498.7601885022317\right.$ i) $\left.\left.\left.\left.\left.\alpha \mathrm{d} 0 \mu \mathrm{r}\right)\right)\right)\right)\right)+\mathrm{k} 1^{3} \mathrm{k}^{2} \alpha \mathrm{d} 0^{2}\left(\left(1.8189894035458565^{*}{ }^{*} \Lambda_{-}\right.\right.$

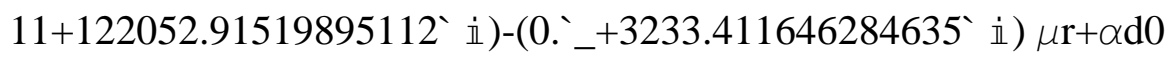
$\left(\left(0 .{ }_{-}+351239.0758260153\right.\right.$ i $)+\mathrm{cr} 0\left(\left(0 ._{-}-76435.51714810776 ` \dot{i}\right)+\alpha \mathrm{d} 0\right.$

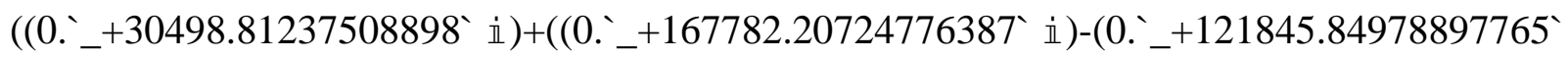
i) $\alpha \mathrm{d} 0) \alpha \mathrm{d} 0))-\left(0 .{ }^{\prime}+36006.25999412406\right.$ i $) \mu \mathrm{r}+\alpha \mathrm{d} 0\left(\left(0 .{ }^{\prime}-504575.19324816315\right.\right.$ ii $)+\left(0 .^{\prime}+65020.645416027124\right.$ i $) \mu \mathrm{r}+\alpha \mathrm{d} 0\left(\left(0 ._{-}-133851.575391237\right.\right.$ i $)-$ $\left(0 ._{-}+25298.164025065093\right.$ i 1$) \mu \mathrm{r}+\alpha \mathrm{d} 0\left(\left(0 .{ }^{\prime}+285452.38361864357\right.\right.$ i $)-$ $\left(0 .{ }_{-}+120317.60600420984\right.$ i $) \alpha \mathrm{d} 0-\left(0 .{ }_{-}+1187.7446277704453 ` \dot{i}\right)$ $\mu \mathrm{r}+\left(0 .^{\prime}+665.0135846696423^{`}\right.$ ii $\left.\left.\left.\left.\left.) \alpha \mathrm{d} 0 \mu \mathrm{r}\right)\right)\right)\right)\right)+\mathrm{k}^{2} \mathrm{k}^{4} \alpha \mathrm{d} 0^{2}\left(\left(-18132.699315044607+0{ }^{`}\right.\right.$ i $)+(212.57068973929498$ _+0. $17627.331456955584 ` 0$. i) $\alpha \mathrm{d} 0))-\left(873.471395590258{ }_{-}+0 . ` \dot{1}\right) \mu \mathrm{r}+\alpha \mathrm{d} 0$ ((199880.1909640432`_+0.`i்)-

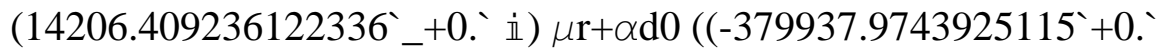
i் $)+\left(5049.861856719788 \_+0\right.$. $\left.\dot{1}\right) \mu \mathrm{r}+\alpha \mathrm{d} 0\left(\left(196216.6394064344 \_+0\right.\right.$.

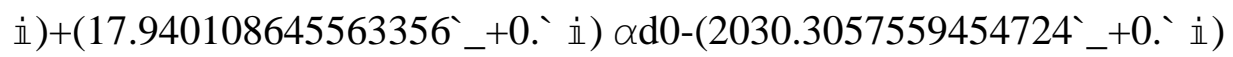

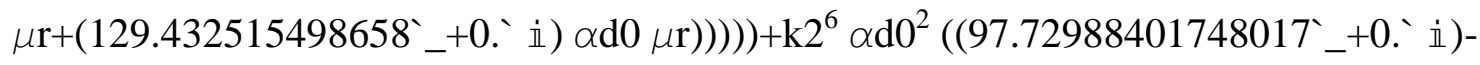
(3.506338886762477_+0`i $) \mu \mathrm{r}+\alpha \mathrm{d} 0\left(\left(16215.36914393136 \_+0 . \grave{i}\right)+\mathrm{cr} 0((-\right.$

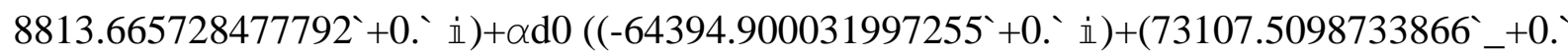
i) $\alpha \mathrm{d} 0))-\left(934.6035434939475 \_+0\right.$. i $) \mu \mathrm{r}+\alpha \mathrm{d} 0$ ((59665.15169273719`_+0.立)(3539.5018891629447_+0.`i $) \mu \mathrm{r}+\alpha \mathrm{d} 0\left(\left(-183786.19497692975+0{ }^{\circ}{ }^{`}\right.\right.$

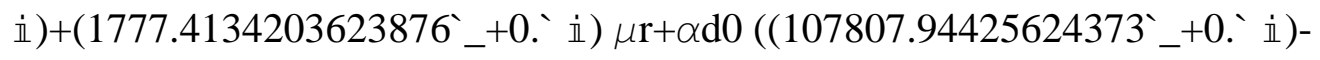

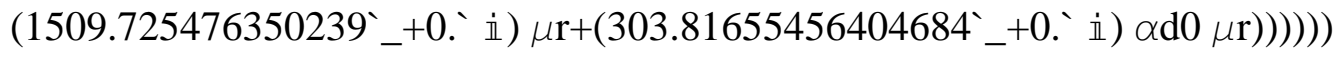

